\title{
Generalized representations of 3-Hom-Lie algebras
}

\author{
S. Mabrouk ${ }^{1}$, A. Makhlouf ${ }^{2}$, S. Massoud $^{3}$ \\ ${ }^{1}$ University of Gafsa, Faculty of Sciences Gafsa, 2112 Gafsa, Tunisia \\ ${ }^{2}$ Université de Haute Alsace, IRIMAS-département de Mathématiques \\ 6, rue des Frères Lumière F-68093 Mulhouse, France \\ ${ }^{3}$ Université de Sfax, Faculté des Sciences, Sfax Tunisia
}

Mabrouksami00@yahoo.fr, Abdenacer.Makhlouf@uha.fr, sonia.massoud2015@gmail.com

Received June 6, 2019

Presented by Consuelo Martínez

Accepted September 30, 2019

Abstract: The propose of this paper is to extend generalized representations of 3-Lie algebras to Hom-type algebras. We introduce the concept of generalized representation of multiplicative 3Hom-Lie algebras, develop the corresponding cohomology theory and study semi-direct products. We provide a key construction, various examples and computation of 2-cocycles of the new cohomology. Also, we give a connection between a split abelian extension of a 3-Hom-Lie algebra and a generalized semidirect product 3-Hom-Lie algebra.

Key words: 3-Hom-Lie algebra, representation, generalized representation, cohomology, abelian extension.

AMS Subject Class. (2010): 17A42, 17B10.

\section{INTRODUCTION}

The first instances of ternary Lie algebras appeared first in Nambu's generalization of Hamiltonian mechanics [23, which was formulated algebraically by Takhtajan [29]. The structure of $n$-Lie algebras was studied by Filippov [15] then completed by Kasymov in [21].

The representation theory of $n$-Lie algebras was first introduced by Kasymov in [21]. The adjoint representation is defined by the ternary bracket in which two elements are fixed. Through fundamental objects one may also represent a 3 -Lie algebra and more generally an $n$-Lie algebra by a Leibniz algebra [11. The cohomology of $n$-Lie algebras, generalizing the ChevalleyEilenberg Lie algebras cohomology, was introduced by Takhtajan 30 in its simplest form, later a complex adapted to the study of formal deformations was introduced by Gautheron [17, then reformulated by Daletskii and Takhtajan [11] using the notion of base Leibniz algebra of an $n$-Lie algebra. In [2, 3], the structure and cohomology of 3-Lie algebras induced by Lie algebras has been investigated. 
The concept of generalized representation of a 3-Lie algebra was introduced by Liu, Makhlouf and Sheng in [19]. They study the corresponding generalized semidirect product 3-Lie algebra and cohomology theory. Furthermore, they describe general abelian extensions of 3-Lie algebras using Maurer-Cartan elements. Non-abelian extensions were explored in [26].

The aim of this paper is to extend the concept of generalized representation of 3-Lie algebras to Hom-type algebras. The notion of Hom-Lie algebras was introduced by Hartwig, Larsson, and Silvestrov in [18] as part of a study of deformations of the Witt and the Virasoro algebras. The $n$-Hom-Lie algebras and various generalizations of $n$-ary algebras were considered in [4]. In a Hom-Lie algebra, the Jacobi identity is twisted by a linear map, called the Hom-Jacobi identity. In particular, representations and cohomologies of HomLie algebras were studied in [25], while the representations and cohomology of $n$-Hom-Lie algebras were first studied in [1].

The paper is organized as follows. In Section 1, we provide some basics about 3-Hom-Lie algebras, representations and cohomology. The second Section includes the new concept of generalized representation of a 3-Hom-Lie algebra, extending to Hom-type algebras the notion and results obtained in [19]. We define a corresponding semi-direct product and provide a twist procedure leading to generalized representations of 3-Hom-Lie algebras starting from generalized representations of 3-Hom-Lie algebras and algebra maps. In Section 3, we construct a new cohomology corresponding to generalized representations and show examples. In the last section we discuss abelian extensions of multiplicative 3 -Hom-Lie algebras. One recovers the results in [19] when the twist map is the identity.

\section{Representations of 3-Hom-Lie Algebras}

The aim of this section is to recall some basics about 3-Lie algebras and 3 -Hom-Lie algebras. We refer mainly to [15] and [4]. In this paper, all vector spaces are considered over a field $\mathbb{K}$ of characteristic 0 .

Definition 1.1. A 3-Lie algebra is a pair $(\mathfrak{g},[\cdot, \cdot, \cdot])$ consisting of a $\mathbb{K}$ vector space $\mathfrak{g}$ and a trilinear skew-symmetric multiplication $[\cdot, \cdot, \cdot]$ satisfying the Filippov-Jacobi identity: for $x, y, z, u, v$ in $\mathfrak{g}$

$$
[u, v,[x, y, z]]=[[u, v, x], y, z]+[x,[u, v, y], z]+[x, y,[u, v, z]] .
$$

In this paper, we are dealing with 3 -Hom-Lie algebras corresponding to the following definition. 
Definition 1.2. A 3 -Hom-Lie algebra is a triple $(\mathfrak{g},[\cdot, \cdot, \cdot], \alpha)$ consisting of a $\mathbb{K}$-vector space $\mathfrak{g}$, a trilinear skew-symmetric multiplication $[\cdot, \cdot, \cdot]$ and an algebra map $\alpha: \mathfrak{g} \rightarrow \mathfrak{g}$ satisfying the Hom-Filippov-Jacobi identity: for $x, y, z, u, v$ in $\mathfrak{g}$

$$
\begin{aligned}
{[\alpha(u), \alpha(v),[x, y, z]]=} & {[[u, v, x], \alpha(y), \alpha(z)]+[\alpha(x),[u, v, y], \alpha(z)] } \\
& +[\alpha(x), \alpha(y),[u, v, z]] .
\end{aligned}
$$

Remark 1.3. There is more general definition of 3-Hom-Lie algebras which are given by a quadruple $\left(\mathfrak{g},[\cdot, \cdot, \cdot], \alpha_{1}, \alpha_{2}\right)$ consisting of a $\mathbb{K}$-vector space $\mathfrak{g}$, two linear maps $\alpha_{1}, \alpha_{2}: \mathfrak{g} \rightarrow \mathfrak{g}$ and a trilinear skew-symmetric multiplication $[\cdot, \cdot, \cdot]$ satisfying the following generalized Hom-Filippov-Jacobi identity: for $x, y, z, u, v$ in $\mathfrak{g}$

$$
\begin{aligned}
{\left[\alpha_{1}(u), \alpha_{2}(v),[x, y, z]\right]=} & {\left[[u, v, x], \alpha_{1}(y), \alpha_{2}(z)\right]+\left[\alpha_{1}(x),[u, v, y], \alpha_{2}(z)\right] } \\
& +\left[\alpha_{1}(x), \alpha_{2}(y),[u, v, z]\right] .
\end{aligned}
$$

We get our class of 3-Hom-Lie algebras when $\alpha_{1}=\alpha_{2}=\alpha$ and where $\alpha$ is an algebra morphism. This kind of algebras are usually called multiplicative 3-Hom-Lie algebras.

Proposition 1.4. Let $(\mathfrak{g},[\cdot, \cdot, \cdot])$ be a 3-Lie algebra and $\alpha: \mathfrak{g} \rightarrow \mathfrak{g}$ be a 3-Lie algebra morphism. Then $\left(\mathfrak{g},[\cdot, \cdot, \cdot]_{\alpha}:=\alpha \circ[\cdot, \cdot, \cdot], \alpha\right)$ is a 3 -Hom-Lie algebra.

Let $(\mathfrak{g},[\cdot, \cdot, \cdot], \alpha)$ be a 3 -Hom-Lie algebra, elements in $\wedge^{2} \mathfrak{g}$ are called fundamental objects of the 3 -Hom-Lie algebra $(\mathfrak{g},[\cdot, \cdot, \cdot], \alpha)$. There is a bilinear operation $[\cdot, \cdot]_{\mathcal{L}}$ on $\wedge^{2} \mathfrak{g}$, which is given by

$$
[X, Y]_{\mathcal{L}}=\left[x_{1}, x_{2}, y_{1}\right] \wedge \alpha\left(y_{2}\right)+\alpha\left(y_{1}\right) \wedge\left[x_{1}, x_{2}, y_{2}\right]
$$

for all $X=x_{1} \wedge x_{2}$ and $Y=y_{1} \wedge y_{2}$, and a linear map $\bar{\alpha}$ on $\wedge^{2} \mathfrak{g}$ defined by $\bar{\alpha}(X)=\alpha\left(x_{1}\right) \wedge \alpha\left(x_{2}\right)$, for simplicity, we will write $\bar{\alpha}(X)=\alpha(X)$. It is well-known that $\left(\wedge^{2} \mathfrak{g},[\cdot, \cdot]_{\mathcal{L}}, \bar{\alpha}\right)$ is a Hom-Leibniz algebra [1, 31].

Definition 1.5. A representation of a 3 -Hom-Lie algebra $(\mathfrak{g},[\cdot, \cdot, \cdot], \alpha)$ on a vector space $V$ with respect to $A \in g l(V)$ is a skew-symmetric linear map $\rho: \wedge^{2} \mathfrak{g} \rightarrow \operatorname{End}(V)$ such that

$$
\begin{aligned}
& \rho\left(\alpha\left(x_{1}\right), \alpha\left(x_{2}\right)\right) \circ A \\
& \rho\left(\alpha\left(x_{1}\right), \alpha\left(x_{2}\right)\right) \rho\left(x_{3}, x_{4}\right)-\rho\left(\alpha\left(x_{3}\right), \alpha\left(x_{4}\right)\right) \rho\left(x_{1}, x_{2}\right) \\
& \quad=\left(\rho\left(\left[x_{1}, x_{2}, x_{3}\right], \alpha\left(x_{4}\right)\right)-\rho\left(\left[x_{1}, x_{2}, x_{4}\right], \alpha\left(x_{3}\right)\right)\right) \circ A
\end{aligned}
$$




$$
\begin{aligned}
& \rho\left(\left[x_{1}, x_{2}, x_{3}\right], \alpha\left(x_{4}\right)\right) \circ A-\rho\left(\alpha\left(x_{2}\right), \alpha\left(x_{3}\right)\right) \rho\left(x_{1}, x_{4}\right) \\
& =\rho\left(\alpha\left(x_{3}\right), \alpha\left(x_{1}\right)\right) \rho\left(x_{2}, x_{4}\right)+\rho\left(\alpha\left(x_{1}\right), \alpha\left(x_{2}\right)\right) \rho\left(x_{3}, x_{4}\right),
\end{aligned}
$$

for $x_{1}, x_{2}, x_{3}$ and $x_{4}$ in $\mathfrak{g}$.

Theorem 1.6. Let $(\mathfrak{g},[\cdot, \cdot, \cdot])$ be a 3 -Lie algebra, $(V, \rho)$ be a representation, $\alpha: \mathfrak{g} \rightarrow \mathfrak{g}$ be a 3-Lie algebra morphism and $A: V \rightarrow V$ be a linear map such that $A \circ \rho\left(x_{1}, x_{2}\right)=\rho\left(\alpha\left(x_{1}\right), \alpha\left(x_{2}\right)\right) \circ A$. Then $(V, \widetilde{\rho}:=A \circ \rho, A)$ is a representation of the 3 -Hom-Lie algebra $\left(\mathfrak{g},[\cdot, \cdot, \cdot]_{\alpha}:=\alpha \circ[\cdot, \cdot, \cdot], \alpha\right)$.

Proof. Let $x_{i} \in \mathfrak{g}$, where $1 \leq i \leq 5$. Then we have

$$
\begin{gathered}
\widetilde{\rho}\left(\left[x_{3}, x_{4}, x_{5}\right]_{\alpha}, \alpha\left(x_{1}\right)\right) \circ A-\widetilde{\rho}\left(\alpha\left(x_{3}\right), \alpha\left(x_{4}\right)\right) \widetilde{\rho}\left(x_{5}, x_{1}\right)-\widetilde{\rho}\left(\alpha\left(x_{4}\right), \alpha\left(x_{5}\right)\right) \widetilde{\rho}\left(x_{3}, x_{1}\right) \\
-\widetilde{\rho}\left(\alpha\left(x_{5}\right), \alpha\left(x_{3}\right)\right) \widetilde{\rho}\left(x_{4}, x_{1}\right) \\
=A^{2} \circ\left(\rho\left(\left[x_{3}, x_{4}, x_{5}\right], x_{1}\right)-\rho\left(x_{3}, x_{4}\right) \rho\left(x_{5}, x_{1}\right)\right. \\
\left.-\rho\left(x_{4}, x_{5}\right) \rho\left(x_{3}, x_{1}\right)-\rho\left(x_{5}, x_{3}\right) \rho\left(x_{4}, x_{1}\right)\right)=0 .
\end{gathered}
$$

The second condition $(1.2)$ is obtained similarly.

The previous result allows to twist along morphisms a 3-Lie algebra with a representation to a 3 -Hom-Lie algebra with a corresponding representation.

Proposition 1.7. Let $(\mathfrak{g},[\cdot, \cdot, \cdot], \alpha)$ be a 3 -Hom-Lie algebra, $V$ be a vector space, $A \in g l(V)$ and $\rho: \wedge^{2} \mathfrak{g} \rightarrow g l(V)$ be a skew-symmetric linear map. Then $(V ; \rho, A)$ is a representation of 3-Hom-Lie algebra $\mathfrak{g}$ if and only if there is a 3 -Hom-Lie algebra structure $\left(\mathfrak{g} \oplus V,[\cdot, \cdot, \cdot]_{\rho}, \alpha_{\mathfrak{g} \oplus V}\right)$ on the direct sum of vector spaces $\mathfrak{g} \oplus V$, defined by

$$
\begin{aligned}
{\left[x_{1}+v_{1}, x_{2}+v_{2}, x_{3}+v_{3}\right]_{\rho}=} & {\left[x_{1}, x_{2}, x_{3}\right]+\rho\left(x_{1}, x_{2}\right) v_{3} } \\
& +\rho\left(x_{3}, x_{1}\right) v_{2}+\rho\left(x_{2}, x_{3}\right) v_{1},
\end{aligned}
$$

and $\alpha_{\mathfrak{g} \oplus V}=\alpha+A$, for all $x_{i} \in \mathfrak{g}, v_{i} \in V, 1 \leq i \leq 3$. The obtained 3-Hom-Lie algebra is denoted by $\mathfrak{g} \ltimes_{\rho} V$ and called semidirect product.

Let $(\mathfrak{g},[\cdot, \cdot, \cdot], \alpha)$ be a 3 -Hom-Lie algebra and $(V, \rho, A)$ be a representation of $\mathfrak{g}$. We denote by $C_{\alpha, A}^{p}(\mathfrak{g}, V)$ the space of all linear maps

$$
\varphi: \underbrace{\wedge^{2} \mathfrak{g} \otimes \cdots \otimes \wedge^{2} \mathfrak{g}}_{(p-1)} \wedge \mathfrak{g} \rightarrow V
$$


satisfying:

$$
A \circ \varphi\left(X_{1} \otimes \cdots \otimes X_{p-1}, y\right)=\varphi\left(\alpha\left(X_{1}\right) \otimes \cdots \otimes \alpha\left(X_{p-1}\right), \alpha(y)\right),
$$

for all $X_{1}, \ldots, X_{p-1} \in \wedge^{2} \mathfrak{g}, y \in \mathfrak{g}$. It is called the space of $p$-cochains.

Let $\varphi$ be a $(p-1)$-cochain, the coboundary operator $\delta_{\rho}: C_{\alpha, A}^{p-1}(\mathfrak{g}, V) \rightarrow$ $C_{\alpha, A}^{p}(\mathfrak{g}, V)$ is given by

$$
\begin{aligned}
\left(\delta_{\rho} \varphi\right)\left(X_{1}, \ldots, X_{p}, z\right) & \\
= & \sum_{1 \leq j<k}(-1)^{j} \varphi\left(\alpha\left(X_{1}\right), \ldots, \hat{X}_{j}, \ldots, \alpha\left(X_{k-1}\right),\left[X_{j}, X_{k}\right]_{\mathcal{L}}, \alpha\left(X_{k+1}\right)\right. \\
& \left.\quad \ldots, \alpha\left(X_{p}\right), \alpha(z)\right) \\
& +\sum_{j=1}^{p}(-1)^{j} \varphi\left(\alpha\left(X_{1}\right), \ldots, \hat{X}_{j}, \ldots, \alpha\left(X_{p}\right),\left[X_{j}, z\right]\right) \\
& +\sum_{j=1}^{p}(-1)^{j+1} \rho\left(\alpha^{p}\left(X_{j}\right)\right) \varphi\left(X_{1}, \ldots, \hat{X}_{j}, \ldots, X_{p}, z\right) \\
& +(-1)^{p+1}\left(\rho\left(\alpha^{p}\left(y_{p}\right), \alpha^{p}(z)\right) \varphi\left(X_{1}, \ldots, X_{p-1}, x_{p}\right)\right. \\
& \left.+\rho\left(\alpha^{p}(z), \alpha^{p}\left(x_{p}\right)\right) \alpha\left(X_{1}, \ldots, X_{p-1}, y_{p}\right)\right)
\end{aligned}
$$

for all $X_{i}=\left(x_{i}, y_{i}\right) \in \wedge^{2} \mathfrak{g}, z \in \mathfrak{g}$ and where $\left[X_{i}, z\right]=\left[x_{i}, y_{i}, z\right]$. An element $\varphi \in C_{\alpha, A}^{p-1}(\mathfrak{g}, V)$ is called a $p$-cocycle if $\delta_{\rho} \varphi=0$. It is called a $p$-coboundary if there exists some $f \in C_{\alpha, A}^{p-2}(\mathfrak{g}, V)$ such that $\varphi=\delta_{\rho} f$. Denote by $Z_{3 H L}^{p}(\mathfrak{g} ; V)$ and $B_{3 H L}^{p}(\mathfrak{g} ; V)$ the sets of $p$-cocycles and $p$-coboundaries respectively. Then the $p$-th cohomology group is

$$
H_{3 H L}^{p}(\mathfrak{g} ; V)=Z_{3 H L}^{p}(\mathfrak{g} ; V) / B_{3 H L}^{p}(\mathfrak{g} ; V) .
$$

In [28], the author constructed a graded Lie algebra structure by which one can describe an $n$-Leibniz algebra structure as a canonical structure. Here, we give the precise formulas for the 3-Hom-Lie algebra case, generalizing the result in [19].

Set $C_{\alpha, \alpha}(\mathfrak{g}, \mathfrak{g})=\oplus_{p \geq 0} C_{\alpha, \alpha}^{p}(\mathfrak{g}, \mathfrak{g})$. Let $\varphi \in C_{\alpha, \alpha}^{q}(\mathfrak{g}, \mathfrak{g}), \quad \psi \in C_{\alpha, \alpha}^{p}(\mathfrak{g}, \mathfrak{g})$, $p, q \geq 0, X_{i}=x_{i} \wedge y_{i} \in \wedge^{2} \mathfrak{g}$ for $i=1,2, \ldots, p+q$ and $x \in \mathfrak{g}$. For each subset $J=\left\{j_{1}, \ldots, j_{p}\right\}_{j_{1}<\cdots<j_{p}} \subset N \triangleq\{1,2, \ldots, p+q\}$, let $I=\left\{i_{1}, \ldots, i_{q}\right\}_{i_{1}<\cdots<i_{q}}$ $=N / J$. Define on the graded vector space $C_{\alpha, \alpha}(\mathfrak{g}, \mathfrak{g})$ the graded commutator bracket

$$
[\varphi, \psi]^{3 H L}=(-1)^{p q} j_{\psi}^{\alpha}(\varphi)-j_{\varphi}^{\alpha}(\psi)=(-1)^{p q} \varphi \circ_{\alpha} \psi-\psi \circ_{\alpha} \varphi
$$


where $\varphi \circ_{\alpha} \psi \in C_{\alpha, \alpha}^{p+q}(\mathfrak{g}, \mathfrak{g})$ is defined by

$$
\begin{aligned}
& j_{\psi}^{\alpha}(\varphi)\left(X_{1}, \ldots, X_{p+q}, x\right)=\varphi \circ_{\alpha} \psi\left(X_{1}, \ldots, X_{p+q}, x\right) \\
& =\sum_{J, j_{q}<i_{k+1} \leq p+q}(-1)^{(J, I)} \varphi\left(\alpha^{p}\left(X_{i_{1}}\right), \ldots, \alpha^{p}\left(X_{i_{k}}\right),\right. \\
& \left.\quad \psi\left(X_{j_{1}}, \ldots, X_{j_{p}},\right) \bullet \alpha X_{i_{k+1}}, \ldots, \alpha^{p}\left(X_{i_{q}}\right), \alpha^{p}(x)\right) \\
& \quad+\sum_{J}(-1)^{(J, I)}(-1)^{q} \varphi\left(\alpha^{p}\left(X_{i_{1}}\right), \ldots, \alpha^{p}\left(X_{i_{q}}\right), \psi\left(X_{j_{1}}, \ldots, X_{j_{p}}, x\right)\right),
\end{aligned}
$$

with

$$
\begin{aligned}
\psi\left(X_{j_{1}}, \ldots, X_{j_{q}},\right) \bullet{ }_{\alpha} X_{i_{k+1}}= & \psi\left(X_{j_{1}}, \ldots, X_{j_{p}}, x_{i_{k+1}}\right) \wedge \alpha^{p}\left(y_{i_{k+1}}\right) \\
& +\alpha^{p}\left(x_{i_{k+1}}\right) \wedge \psi\left(X_{j_{1}}, \ldots, X_{j_{p}}, y_{i_{k+1}}\right)
\end{aligned}
$$

where $k$ is uniquely determined by the condition $j_{p} \leq i_{k+1}$ and if $j_{p} \leq i_{1}$ then $j_{p}=p, i_{1}=p+1$ and $(-1)^{(J, I)}$ is the sign of the permutation $(J, I)=$ $\left(j_{1}, \ldots, j_{p}, i_{1}, \ldots, i_{q}\right)$ of $N$.

We need the following lemma to establish a structure of graded Lie algebra on $C_{\alpha, \alpha}(\mathfrak{g}, \mathfrak{g})$.

Lemma 1.8. We have $j_{[\varphi, \psi]^{3 H L}}=-\left[j_{\varphi}^{\alpha}, j_{\psi}^{\alpha}\right]$ for all $\varphi, \psi \in C_{\alpha, \alpha}(\mathfrak{g}, \mathfrak{g})$, where $[\cdot, \cdot]$ is the graded commutator on $\operatorname{End}\left(C_{\alpha, \alpha}(\mathfrak{g}, \mathfrak{g})\right)$.

Proof. Let $\varphi \in C_{\alpha, \alpha}^{q}(\mathfrak{g}, \mathfrak{g}), \psi \in C_{\alpha, \alpha}^{p}(\mathfrak{g}, \mathfrak{g}), \xi \in C_{\alpha, \alpha}^{r}(\mathfrak{g}, \mathfrak{g}), X_{1}, \ldots, X_{p+q+r} \in$ $\wedge^{2} \mathfrak{g}$ and $x \in \mathfrak{g}$

$$
\begin{aligned}
{\left[j_{\varphi}^{\alpha}, j_{\psi}^{\alpha}\right](\xi)\left(X_{1}, X_{2}, \ldots, X_{p+q+r}, x\right) } & \\
& =\left(j_{\varphi}^{\alpha}\left(j_{\psi}^{\alpha} \xi\right)-(-1)^{p q} j_{\psi}^{\alpha}\left(j_{\varphi}^{\alpha} \xi\right)\right)\left(X_{1}, X_{2}, \ldots, X_{p+q+r}, x\right) \\
& =D_{1}-(-1)^{p q} D_{2},
\end{aligned}
$$

where

$$
\begin{aligned}
& D_{1}=j_{\varphi}^{\alpha}\left(j_{\psi}^{\alpha} \xi\right)\left(X_{1}, X_{2}, \ldots, X_{p+q+r}, x\right), \\
& \left.D_{2}=j_{\psi}^{\alpha}\left(j_{\varphi}^{\alpha} \xi\right)\right)\left(X_{1}, X_{2}, \ldots, X_{p+q+r}, x\right) .
\end{aligned}
$$

For each subset $J=\left\{j_{1}, \ldots, j_{q}\right\}_{j_{1}<\cdots<j_{q}} \subset N \triangleq\{1,2, \ldots, p+q+r\}$, let $I=\left\{i_{1}, \ldots, i_{p+r}\right\}_{i_{1}<\cdots<i_{p+r}}=N \backslash J$ and $L=\left\{l_{1}, \ldots, l_{p}\right\}_{l_{1}<\cdots<l_{p}} \subset I \triangleq$ $\left\{i_{1}, i_{2}, \ldots, i_{p+r}\right\}$, let $H=\left\{h_{1}, \ldots, h_{r}\right\}_{h_{1}<\cdots<h_{r}}=I \backslash L$. 
We have for $D_{1}$ :

$$
\begin{aligned}
& j_{\varphi}^{\alpha}\left(j_{\psi}^{\alpha} \xi\right)\left(X_{1}, X_{2}, \ldots, X_{p+q+r}, x\right)=\left(j_{\psi}^{\alpha}(\xi)\right) \circ_{\alpha} \varphi\left(X_{1}, X_{2}, \ldots, X_{p+q+r}, x\right) \\
& =\sum_{J, j_{q}<i_{k+1} \leq p+q+r}(-1)^{(J, I)}\left(j_{\psi}^{\alpha}(\xi)\right)\left(\alpha^{q}\left(X_{i_{1}}\right), \ldots, \alpha^{q}\left(X_{i_{k}}\right), \varphi\left(X_{j_{1}}, \ldots, X_{j_{q}},\right) \bullet{ }_{\alpha} X_{i_{k+1}}, \alpha^{q}\left(X_{i_{k+2}}\right), \ldots, \alpha^{q}\left(X_{i_{p+r}}\right), \alpha^{q}(x)\right) \\
& +\sum_{J}(-1)^{(J, I)}(-1)^{p+r}\left(j_{\psi}^{\alpha}(\xi)\right)\left(\alpha^{q}\left(X_{i_{1}}\right), \ldots, \alpha^{q}\left(X_{i_{p+r}}\right), \varphi\left(X_{j_{1}}, \ldots, X_{j_{q}}, x\right)\right) \\
& =\sum_{J, j_{q}<i_{k+1} \leq p+q+r} \sum_{\substack{l_{l} l_{p}<h_{m+1} \leq i_{p+r}, i_{k+1}=h_{n}, n \leq m}}(-1)^{(J, I)}(-1)^{(L, H)} \xi\left(\alpha^{p+q}\left(X_{h_{1}}\right), \ldots, \alpha^{p+q}\left(X_{h_{n-1}}\right), \alpha^{p}\left(\varphi\left(X_{j_{1}}, \ldots, X_{j_{q}},\right) \bullet_{\alpha} X_{i_{k+1}}\right),\right. \\
& \left.\ldots, \alpha^{p+q}\left(X_{h_{m}}\right), \psi\left(\alpha^{q}\left(X_{l_{1}}\right), \ldots, \alpha^{q}\left(X_{l_{p}}\right),\right) \bullet \alpha \alpha^{q}\left(X_{h_{m+1}}\right), \alpha^{p+q}\left(X_{h_{m+2}}\right), \ldots, \alpha^{p+q}\left(X_{h_{r}}\right), \alpha^{p+q}(x)\right) \\
& +\sum_{J, j_{q}<i_{k+1} \leq p+q+r} \sum_{\substack{L, l_{p}<h_{m+1} \leq i_{p+r}, i_{k+1}=h_{m+1}}}(-1)^{(J, I)}(-1)^{(L, H)} \xi\left(\alpha^{p+q}\left(X_{h_{1}}\right), \ldots, \alpha^{p+q}\left(X_{h_{m}}\right), \psi\left(\alpha^{q}\left(X_{l_{1}}\right), \ldots, \alpha^{q}\left(X_{l_{p}}\right),\right)\right. \\
& \left.\bullet_{\alpha}\left(\varphi\left(X_{j_{1}}, \ldots, X_{j_{q}},\right) \bullet_{\alpha} X_{i_{k+1}}\right), \alpha^{p+q}\left(X_{h_{m+2}}\right), \ldots, \alpha^{p+q}\left(X_{h_{r}}\right), \alpha^{p+q}(x)\right) \\
& +\sum_{J, j_{q}<i_{k+1} \leq p+q+r} \sum_{\substack{L, l_{p}<h_{m+1} \leq i_{p+r}, i_{k+1}=h_{n}, n>m+1}}(-1)^{(J, I)}(-1)^{(L, H)} \xi\left(\alpha^{p+q}\left(X_{h_{1}}\right), \ldots, \alpha^{p+q}\left(X_{h_{m}}\right), \psi\left(\alpha^{q}\left(X_{l_{1}}\right), \ldots, \alpha^{q}\left(X_{l_{p}}\right),\right)\right. \\
& \bullet_{\alpha} \alpha^{p+q}\left(X_{h_{m+1}}\right), \alpha^{p+q}\left(X_{h_{m+2}}\right), \ldots, \alpha^{p+q}\left(X_{h_{n-1}}\right), \alpha^{p}\left(\varphi\left(X_{j_{1}}, \ldots, X_{j_{q}},\right)\right. \\
& \text { - } \left.\alpha X_{i_{k+1}}\right), \alpha^{p+q}\left(X_{h_{n+1}}, \ldots, \alpha^{p+q}\left(X_{h_{r}}\right), \alpha^{p+q}(x)\right)
\end{aligned}
$$




$$
\begin{aligned}
& +\sum_{J, j_{q}<i_{k+1} \leq p+q+r} \sum_{\substack{l_{l_{p}<h_{m+1} \leq i_{p+r}}, i_{k+1}=l_{s}, s \leq p}}(-1)^{(J, I)}(-1)^{(L, H)} \xi\left(\alpha^{p+q}\left(X_{h_{1}}\right), \ldots, \alpha^{p+q}\left(X_{h_{m}}\right), \psi\left(\alpha^{q}\left(X_{l_{1}}\right), \ldots, \alpha^{q}\left(X_{l_{s-1}}\right),\right.\right. \\
& \left.\left.\varphi\left(X_{j_{1}}, \ldots, X_{j_{q}}, X_{j_{q}},\right) \bullet_{\alpha} X_{i_{k+1}}, \alpha^{q}\left(X_{l_{s+1}}\right), \ldots, \alpha^{q}\left(X_{l_{p}}\right),\right) \bullet_{\alpha} \alpha^{q}\left(X_{h_{m+1}}\right), \alpha^{p+q}\left(X_{h_{m+2}}\right), \ldots, \alpha^{p+q}\left(X_{h_{r}}\right), \alpha^{p+q}(x)\right) \\
& +\sum_{J, j_{q}<i_{k+1} \leq p+q+r} \sum_{L, i_{k+1}=h_{n}}(-1)^{(J, I)}(-1)^{(L, H)}(-1)^{r} \xi\left(\alpha^{p+q}\left(X_{h_{1}}\right), \ldots, \alpha^{p+q}\left(X_{h_{n-1}}\right), \alpha^{p}\left(\varphi\left(X_{j_{1}}, \ldots, X_{j_{q}},\right) \bullet_{\alpha} X_{i_{k+1}}\right),\right. \\
& \left.\alpha^{p+q}\left(X_{h_{n+1}}\right), \ldots, \alpha^{p+q}\left(X_{h_{r}}\right), \psi\left(\alpha^{q}\left(X_{l_{1}}\right), \ldots, \alpha^{q}\left(X_{l_{p}}\right), \alpha^{q}(x)\right)\right) \\
& +\sum_{J, j_{q}<i_{k+1} \leq p+q+r} \sum_{L, i_{k+1}=l_{s}}(-1)^{(J, I)}(-1)^{(L, H)}(-1)^{r} \xi\left(\alpha^{p+q}\left(X_{h_{1}}\right), \ldots, \alpha^{p+q}\left(X_{h_{r}}\right), \psi\left(\alpha^{q}\left(X_{l_{1}}\right), \ldots, \alpha^{q}\left(X_{l_{s-1}}\right),\right.\right. \\
& \left.\left.\varphi\left(X_{j_{1}}, \ldots, X_{j_{q}},\right) \bullet_{\alpha} X_{i_{k+1}}, \alpha^{q}\left(X_{l_{s+1}}\right), \ldots, \alpha^{q}\left(X_{l_{p}}\right), \alpha^{q}(x)\right)\right) \\
& +\sum_{J} \sum_{L, l_{p}<h_{m+1} \leq i_{p+r}}(-1)^{(J, I)}(-1)^{p+r}(-1)^{(L, H)} \xi\left(\alpha^{p+q}\left(X_{h_{1}}\right), \ldots, \alpha^{p+q}\left(X_{h_{m}}\right), \psi\left(\alpha^{q}\left(X_{l_{1}}\right), \ldots, \alpha^{q}\left(X_{l_{p}}\right),\right) \bullet \bullet_{\alpha}\left(\alpha^{q}\left(X_{h_{m+1}}\right),\right.\right. \\
& \left.\alpha^{p+q}\left(X_{h_{m+2}}\right), \ldots, \alpha^{p+q}\left(X_{h_{r}}\right), \alpha^{p}\left(\varphi\left(X_{j_{1}}, \ldots, X_{j_{q}}, x\right)\right)\right) \\
& +\sum_{J} \sum_{L}(-1)^{(J, I)}(-1)^{p+r}(-1)^{(L, H)}(-1)^{r} \xi\left(\alpha^{p+q}\left(X_{h_{1}}\right), \ldots, \alpha^{p+q}\left(X_{h_{r}}\right), \psi\left(\alpha^{q}\left(X_{l_{1}}\right), \ldots, \alpha^{p+q}\left(X_{l_{p}}\right), \varphi\left(X_{j_{1}}, \ldots, X_{j_{q}}, x\right)\right)\right) \text {. }
\end{aligned}
$$

Similarly one can compute $D_{2}$. 
Let $A=\left\{a_{1}, a_{2}, \ldots, a_{p+q}\right\}_{a_{1}<a_{2}<\cdots<a_{p+q}} \subseteq N=\{1, \ldots, p+q+r\}, H=\left\{h_{1}, \ldots, h_{r}\right\}_{h_{1}<h_{2}<\cdots<h_{r}}=N \backslash A$, and $J=\left\{j_{1}, \ldots, j_{q}\right\}_{j_{1}<\cdots<j_{q}} \subseteq A$ and $L=\left\{l_{1}, \ldots, l_{p}\right\}_{l_{1}<\cdots<l_{p}}=A \backslash J$. We have:

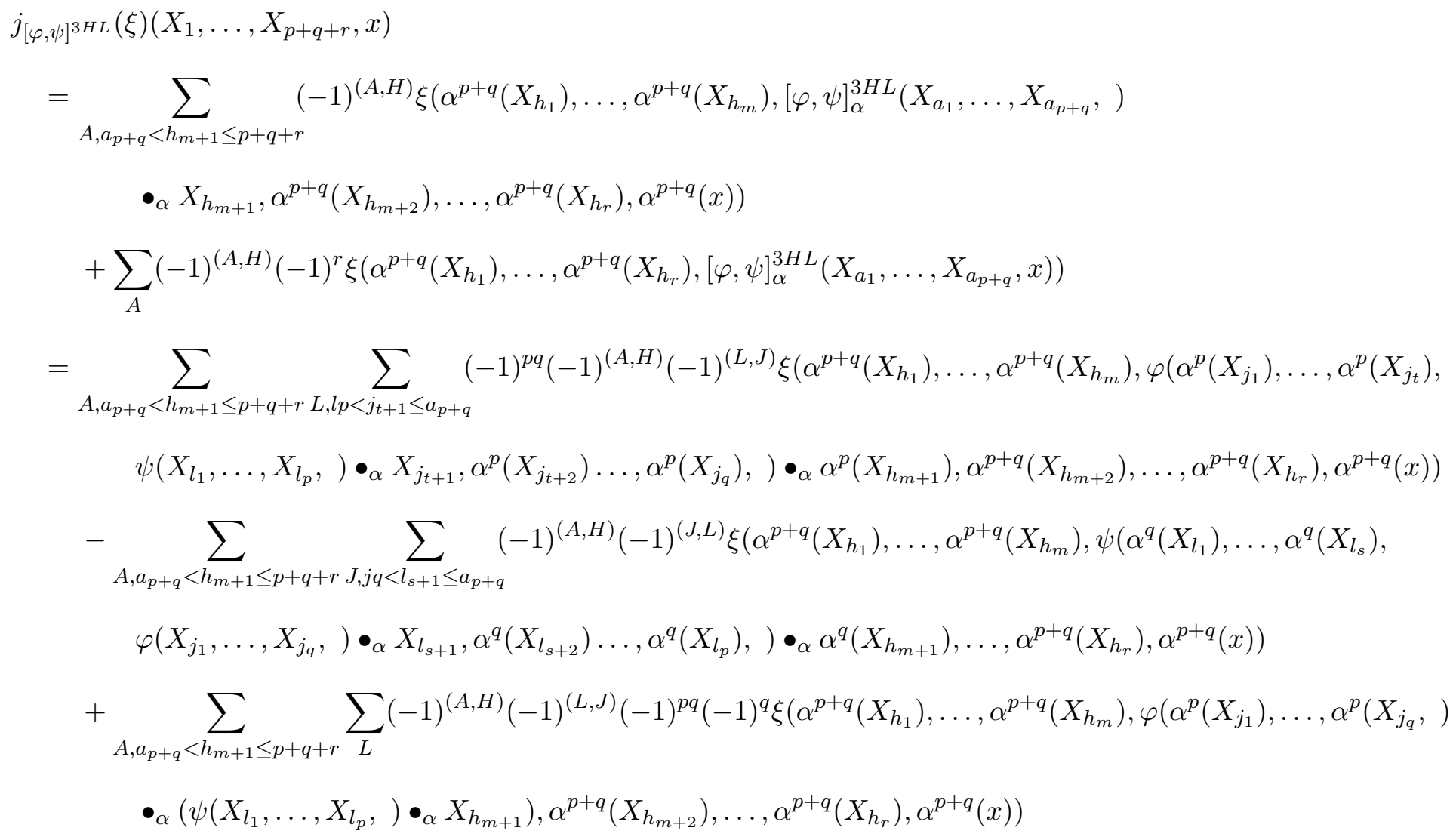




$$
\begin{aligned}
& -\sum_{A, a_{p+q}<h_{m+1} \leq p+q+r} \sum_{J}(-1)^{(A, H)}(-1)^{(J, L)}(-1)^{p q}(-1)^{P} \xi\left(\alpha^{p+q}\left(X_{h_{1}}\right), \ldots, \alpha^{p+q}\left(X_{h_{m}}\right), \psi\left(\alpha^{q}\left(X_{l_{1}}\right), \ldots, \alpha^{q}\left(X_{l_{p}},\right)\right.\right. \\
& \left.\bullet_{\alpha}\left(\varphi\left(X_{j_{1}}, \ldots, X_{j_{q}},\right) \bullet_{\alpha} X_{h_{m+1}}\right), \alpha^{p+q}\left(X_{h_{m+2}}\right), \ldots, \alpha^{p+q}\left(X_{h_{r}}\right), \alpha^{p+q}(x)\right) \\
& +\sum_{A} \sum_{L, l_{p}<j_{t+1} \leq a_{p+q}}(-1)^{r}(-1)^{p q}(-1)^{(A, H)}(-1)^{(L, J)}\left(\xi \left(\alpha^{p+q}\left(X_{h_{1}}\right), \ldots, \alpha^{p+q}\left(X_{h_{r}}\right), \varphi\left(\alpha^{p}\left(X_{j_{1}}\right), \ldots, \alpha^{p}\left(X_{j_{t}}\right),\right.\right.\right. \\
& \left.\left.\psi\left(X_{l_{1}}, \ldots, X_{l_{p}},\right) \bullet \alpha X_{j_{t+1}}, \alpha^{p}\left(X_{j_{t+2}}\right), \ldots, \alpha^{p}\left(X_{j_{q}}\right), \alpha^{p}(x)\right)\right) \\
& +\sum_{A} \sum_{L}(-1)^{r}(-1)^{q}(-1)^{p q}(-1)^{(A, H)}(-1)^{(L, J)}\left(\xi \left(\alpha^{p+q}\left(X_{h_{1}}\right), \ldots, \alpha^{p+q}\left(X_{h_{r}}\right), \varphi\left(\alpha^{p}\left(X_{j_{1}}\right), \ldots, \alpha^{p}\left(X_{j_{q}}\right), \psi\left(X_{l_{1}}, \ldots, X_{l_{p}}, x\right)\right)\right.\right. \\
& -\sum_{A} \sum_{J, J_{q}<l_{s+1} \leq a_{p+q}}(-1)^{r}(-1)^{(A, H)}(-1)^{(J, L)}\left(\xi \left(\alpha^{p+q}\left(X_{h_{1}}\right), \ldots, \alpha^{p+q}\left(X_{h_{r}}\right), \psi\left(\alpha^{q}\left(X_{l_{1}}\right), \ldots, \alpha^{q}\left(X_{l_{s}}\right)\right.\right.\right. \\
& \left.\left.\varphi\left(X_{j_{1}}, \ldots, X_{j_{q}},\right) \bullet \alpha X_{l_{s+1}}, \alpha^{q}\left(X_{l_{s+2}}\right), \ldots, \alpha^{q}\left(X_{l_{p}}\right), \alpha^{q}(x)\right)\right) \\
& -\sum_{A} \sum_{J}(-1)^{p}(-1)^{r}(-1)^{(A, H)}(-1)^{(J, L)}\left(\xi \left(\alpha^{p+q}\left(X_{h_{1}}\right), \ldots, \alpha^{p+q}\left(X_{h_{r}}\right), \psi\left(\alpha^{q}\left(X_{l_{1}}\right), \ldots, \alpha^{q}\left(X_{l_{p}}\right), \varphi\left(X_{j_{1}}, \ldots, X_{j_{q}}, x\right)\right) .\right.\right.
\end{aligned}
$$


THEOREM 1.9. The pair $\left(C_{\alpha, \alpha}(\mathfrak{g}, \mathfrak{g}),[\cdot, \cdot]^{3 H L}\right)$ is a graded Lie algebra.

Proof. Let $\varphi \in C_{\alpha, \alpha}^{q}(\mathfrak{g}, \mathfrak{g}), \psi \in C_{\alpha, \alpha}^{p}(\mathfrak{g}, \mathfrak{g})$ and $\phi \in C_{\alpha, \alpha}^{r}(\mathfrak{g}, \mathfrak{g})$.

(1) Skew-symmetry:

$$
\begin{aligned}
{[\varphi, \psi]^{3 H L} } & =(-1)^{p q} j_{\varphi}^{\alpha}(\psi)-j_{\psi}^{\alpha}(\varphi) \\
& =(-1)^{p q+1}\left((-1)^{p q} j_{\psi}^{\alpha}(\varphi)-j_{\varphi}^{\alpha}(\psi)\right) \\
& =-(-1)^{p q}[\psi, \varphi]^{3 H L} .
\end{aligned}
$$

(2) Graded Jacobi identity:

$$
\begin{aligned}
\circlearrowleft_{\varphi, \psi, \phi}(-1)^{q r}[\varphi, & {\left.[\psi, \phi]^{3 H L}\right]^{3 H L} } \\
= & (-1)^{q p} j_{[\psi, \phi]^{3 H L}}(\varphi)-(-1)^{q r} j_{\varphi}^{\alpha}\left([\psi, \phi]^{3 H L}\right) \\
& +(-1)^{p r} j_{[\phi, \varphi]^{3 H L}}(\psi)-(-1)^{p q} j_{\psi}^{\alpha}\left([\phi, \varphi]^{3 H L}\right) \\
& +(-1)^{r q} j_{[\varphi, \psi]^{3 H L}}(\phi)-(-1)^{r p} j_{\phi}^{\alpha}\left([\varphi, \psi]^{3 H L}\right) \\
= & (-1)^{q p} j_{[\psi, \phi]^{3 H L}}(\varphi)-(-1)^{q r} j_{\varphi}^{\alpha}\left((-1)^{r p} j_{\phi}^{\alpha}(\psi)-j_{\psi}^{\alpha}(\phi)\right) \\
& +(-1)^{p r} j_{[\phi, \varphi]^{3 H L}}(\psi)-(-1)^{p q} j_{\psi}^{\alpha}\left((-1)^{q r} j_{\varphi}^{\alpha}(\phi)-j_{\phi}^{\alpha}(\varphi)\right) \\
& +(-1)^{r q} j_{[\varphi, \psi]^{3 H L}}(\phi)-(-1)^{r p} j_{\phi}^{\alpha}\left((-1)^{q p} j_{\psi}^{\alpha}(\varphi)-j_{\varphi}^{\alpha}(\psi)\right) .
\end{aligned}
$$

Organizing these terms leads to

$$
\begin{aligned}
& \circlearrowleft_{\varphi, \psi, \phi}(-1)^{q r}\left[\varphi,[\psi, \phi]^{3 H L}\right]^{3 H L} \\
&=(-1)^{p q} j_{[\psi, \phi]^{3 H L}}(\varphi)+(-1)^{p q}\left(j_{\psi}^{\alpha}\left(j_{\phi}^{\alpha}(\varphi)\right)-(-1)^{r p} j_{\phi}^{\alpha}\left(j_{\psi}^{\alpha}(\varphi)\right)\right) \\
&+(-1)^{r p} j_{[\phi, \varphi]^{3 H L}}(\psi)+(-1)^{r p}\left(j_{\phi}^{\alpha}\left(j_{\varphi}^{\alpha}(\psi)\right)-(-1)^{q r} j_{\varphi}^{\alpha}\left(j_{\phi}^{\alpha}(\psi)\right)\right) \\
&+(-1)^{q r} j_{[\varphi, \psi]^{3 H L}}(\phi)+(-1)^{q r}\left(j_{\varphi}^{\alpha}\left(j_{\psi}^{\alpha}(\phi)\right)-(-1)^{q p} j_{\psi}^{\alpha}\left(j_{\varphi}^{\alpha}(\phi)\right)\right) \\
&=(-1)^{p q}\left(\left[j_{\psi}^{\alpha}, j_{\phi}^{\alpha}\right]+j_{[\psi, \phi]^{3 H L}}\right)(\varphi) \\
&+(-1)^{r p}\left(\left[j_{\phi}^{\alpha}, j_{\varphi}^{\alpha}\right]+j_{[\phi, \varphi]^{3 H L}}\right)(\psi) \\
&+(-1)^{q r}\left(\left[j_{\varphi}^{\alpha}, j_{\psi}^{\alpha}\right]+j_{[\varphi, \psi]^{3 H L}}\right)(\phi) .
\end{aligned}
$$

Using the previous lemma, we get

$$
\circlearrowleft_{\varphi, \psi, \phi}(-1)^{q r}\left[\varphi,[\psi, \phi]^{3 H L}\right]^{3 H L}=0 .
$$


Remark 1.10. The pair $\left(C_{\alpha, \alpha}(\mathfrak{g}, \mathfrak{g}), \circ_{\alpha}\right)$ is a right symmetric graded algebra.

The previous structure of graded Lie algebra is useful to describe 3-HomLie algebra structures as well as coboundary operators.

Corollary 1.11. The maps $\pi: \wedge^{3} \mathfrak{g} \rightarrow \mathfrak{g}$ and $\alpha: \mathfrak{g} \rightarrow \mathfrak{g}$ define a 3-HomLie structure if and only if $[\pi, \pi]^{3 H L}=0$. by

Let $\mathfrak{g}$ be a 3-Hom-Lie algebra. Given $x_{1}, x_{2} \in \mathfrak{g}$, define $a d: \wedge^{2} \mathfrak{g} \rightarrow g l(\mathfrak{g})$

$$
a d_{x_{1}, x_{2}} y=\left[x_{1}, x_{2}, y\right]
$$

Then, the pair $(a d, \alpha)$ defines a representation of the 3-Hom-Lie algebra $\mathfrak{g}$ on itself, which we call adjoint representation of $\mathfrak{g}$. The coboundary operator associated to this representation is denoted by $\delta_{\mathfrak{g}}$.

Corollary 1.12. If $\pi: \wedge^{3} \mathfrak{g} \rightarrow \mathfrak{g}$ is a 3 -Hom-Lie bracket, then we have

$$
[\pi, \varphi]^{3 H L}=\delta_{g}(\varphi) \quad \text { for all } \varphi \in C_{\alpha, \alpha}^{p}(\mathfrak{g}, \mathfrak{g}), p \geq 0 .
$$

\section{Generalized Representations of 3-Hom-Lie algebras}

In this section, we provide the Hom-type version of generalized representations of a 3-Lie algebras introduced in [19]. First, we show that a representation of a 3-Hom-Lie algebra will give rise to a canonical structure.

Let $\mathfrak{g}$ be a 3-Hom-Lie algebra and $V$ be a vector space. Let $\rho: \wedge^{2} \mathfrak{g} \rightarrow g l(V)$ be a linear map. Then, it induces a linear map $\bar{\rho}: \wedge^{3}(\mathfrak{g} \oplus V) \rightarrow \mathfrak{g} \oplus V$ defined by

$$
\bar{\rho}(x+u, y+v, z+w)=\rho(x, y)(w)+\rho(y, z)(u)+\rho(z, x)(v)
$$

for all $x, y, z \in \mathfrak{g}, u, v, w \in V$. Consider the graded Lie algebra given in Theorem 1.9 associated to the vector space $\mathfrak{g} \oplus V$.

Proposition 2.1. A linear map $\rho: \wedge^{2} \mathfrak{g} \rightarrow g l(V)$ is a representation on a vector space $V$ of the 3 -Hom-Lie algebra $\mathfrak{g}$ with respect to $A \in g l(V)$ if and only if $\pi+\bar{\rho}$ is a canonical structure in the graded Lie algebra associated to $\mathfrak{g} \oplus V$, i.e.

$$
[\pi+\bar{\rho}, \pi+\bar{\rho}]^{3 H L}=0
$$


Proof. By Proposition 1.7, $\rho: \wedge^{2} \mathfrak{g} \rightarrow g l(V)$ is a representation of $\mathfrak{g}$ if and only if $\mathfrak{g} \oplus V$ is a 3-Hom-Lie algebra, where the 3-Hom-Lie structure is exactly given by

$$
\begin{aligned}
{[x+u, y+v, z+w]_{\rho} } & =[x, y, z]+\rho(x, y)(w)+\rho(y, z)(u)+\rho(z, x)(v) \\
& =(\pi+\bar{\rho})(x+u, y+v, z+w),
\end{aligned}
$$

and $\alpha_{\mathfrak{g} \oplus V}=\alpha+A$. Thus, by Lemma 1.11, $\rho: \wedge^{2} \mathfrak{g} \rightarrow g l(V)$ is a representation of $\mathfrak{g}$ if and only if $\pi+\bar{\rho}$ is a canonical structure.

The concept of representation of 3-Lie algebras introduced by Liu, Makhlouf and Sheng [19] is generalized to Hom-type algebras as follows.

Definition 2.2. A generalized representation of a 3-Hom-Lie algebra $(\mathfrak{g},[\cdot, \cdot, \cdot], \alpha)$ with respect to $A \in g l(V)$ consists of linear maps $\rho: \wedge^{2} \mathfrak{g} \rightarrow g l(V)$, $\nu: \mathfrak{g} \rightarrow \operatorname{Hom}\left(\wedge^{2} V, V\right)$, such that

$$
[\pi+\bar{\rho}+\bar{\nu}, \pi+\bar{\rho}+\bar{\nu}]^{3 H L}=0,
$$

where $\bar{\nu}: \wedge^{3}(\mathfrak{g} \oplus V) \rightarrow(\mathfrak{g} \oplus V)$ is induced by $\nu$ via

$$
\bar{\nu}(x+u, y+v, z+w)=\nu(x)(v \wedge w)+\nu(y)(w \wedge u)+\nu(z)(u \wedge v)
$$

for all $x, y, z \in \mathfrak{g}, u, v, w \in V$. We will refer to a generalized representation by $(V ; \rho, \nu, A)$.

Remark 2.3. If $\nu=0$, then we recover the usual definition of a representation of a 3-Hom-Lie algebra on a vector space $V$. If the dimension of the vector space $V$ is 1 , then $\nu$ must be zero. In this case, we only have the usual representation.

Given linear maps $\rho: \wedge^{2} \mathfrak{g} \rightarrow \operatorname{End}(V), \nu: \mathfrak{g} \rightarrow \operatorname{Hom}\left(\wedge^{2} V, V\right)$, and $A: V \rightarrow$ $V$, define a trilinear bracket operation on $\mathfrak{g} \oplus V$ by

$$
\begin{aligned}
{[x+u, y+v, z+w]_{(\rho, \nu)}=} & {[x, y, z]+\rho(x, y)(w)+\rho(y, z)(u) } \\
& +\rho(z, x)(v)+\nu(x)(v \wedge w) \\
& +\nu(y)(w \wedge u)+\nu(z)(u \wedge v) .
\end{aligned}
$$

Theorem 2.4. Let $(\mathfrak{g},[\cdot, \cdot, \cdot], \alpha)$ be a 3-Hom-Lie algebra and $(V ; \rho, \nu, A)$ a generalized representation of $\mathfrak{g}$ with respect to $A$. Then $\left(\mathfrak{g} \oplus V,[\cdot, \cdot, \cdot]_{(\rho, \nu)}\right.$, $\left.\alpha_{\mathfrak{g} \oplus V}=\alpha+A\right)$ is 3 -Hom-Lie algebra, where $[\cdot, \cdot, \cdot]_{(\rho, \nu)}$ is given by (2.1). We call the 3 -Hom-Lie algebra $\left(\mathfrak{g} \oplus V,[\cdot, \cdot, \cdot]_{(\rho, \nu)}, \alpha_{\mathfrak{g} \oplus V}=\alpha+A\right)$ the generalized semidirect product of $\mathfrak{g}$ and $V$. 
Proof. It follows from $[x+u, y+v, z+w](\rho, \nu)=(\pi+\bar{\rho}+\bar{\nu})(x+u, y+v, z+w)$ and Lemma 1.11 .

In the following, we give a characterization of a generalized representation of a 3-Hom-Lie algebra.

Proposition 2.5. Let $\rho: \wedge^{2} \mathfrak{g} \rightarrow \operatorname{End}(V), \nu: \mathfrak{g} \rightarrow \operatorname{Hom}\left(\wedge^{2} V, V\right)$ and $A: V \rightarrow V$ be linear maps. They give rise to a generalized representation of a 3 -Hom-Lie algebra $\mathfrak{g}$ with respect to $A$ if and only if for all $x_{i} \in \mathfrak{g}, v_{j} \in V$, the following equalities hold:

$$
\begin{aligned}
\rho\left(\alpha\left(x_{1}\right), \alpha\left(x_{2}\right)\right) \rho\left(x_{3}, x_{4}\right)= & \rho\left(\left[x_{1}, x_{2}, x_{3}\right], \alpha\left(x_{4}\right)\right) \circ A \\
& -\rho\left(\left[x_{1}, x_{2}, x_{4}\right], \alpha\left(x_{3}\right)\right) \circ A \\
& +\rho\left(\alpha\left(x_{3}\right), \alpha\left(x_{4}\right)\right) \rho\left(x_{1}, x_{2}\right), \\
\rho\left(\left[x_{1}, x_{2}, x_{3}\right], \alpha\left(x_{4}\right)\right) \circ A=\rho & \left.\alpha\left(x_{2}\right), \alpha\left(x_{3}\right)\right) \rho\left(x_{1}, x_{4}\right) \\
& +\rho\left(\alpha\left(x_{3}\right), \alpha\left(x_{1}\right)\right) \rho\left(x_{2}, x_{4}\right) \\
& +\rho\left(\alpha\left(x_{1}\right), \alpha\left(x_{2}\right)\right) \rho\left(x_{3}, x_{4}\right), \\
\rho\left(\alpha\left(x_{1}\right), \alpha\left(x_{2}\right)\right) \nu\left(x_{3}\right)\left(v_{1}, v_{2}\right)= & \left(\left[x_{1}, x_{2}, x_{3}\right]\right)\left(A\left(v_{1}\right), A\left(v_{2}\right)\right) \\
& +\nu\left(\alpha\left(x_{3}\right)\right)\left(\rho\left(x_{1}, x_{2}\right) v_{1}, A\left(v_{2}\right)\right) \\
& +\nu\left(\alpha\left(x_{3}\right)\right)\left(A\left(v_{2}\right), \rho\left(x_{1}, x_{2}\right) v_{1}\right), \\
\nu\left(\alpha\left(x_{1}\right)\right)\left(A\left(v_{1}\right), \rho\left(x_{2}, x_{3}\right) v_{2}\right)= & \nu\left(\alpha\left(x_{3}\right)\right)\left(A\left(v_{2}\right), \rho\left(x_{2}, x_{1}\right) v_{1}\right) \\
& +\nu\left(\alpha\left(x_{2}\right)\right)\left(\rho\left(x_{3}, x_{1}\right) v_{1}, A\left(v_{2}\right)\right) \\
& +\rho\left(\alpha\left(x_{2}\right), \alpha\left(x_{3}\right)\right) \nu\left(x_{1}\right)\left(v_{1}, v_{2}\right), \\
\nu\left(\alpha\left(x_{1}\right)\right)\left(A\left(v_{1}\right), \nu\left(x_{2}\right)\left(v_{2}, v_{3}\right)\right)= & \nu\left(\alpha\left(x_{2}\right)\right)\left(\nu\left(x_{1}\right)\left(v_{1}, v_{2}\right), A\left(v_{3}\right)\right) \\
& +\nu\left(\alpha\left(x_{2}\right)\right)\left(A\left(v_{2}\right), \nu\left(x_{1}\right)\left(v_{1}, v_{3}\right)\right), \\
\nu\left(\alpha\left(x_{1}\right)\right)\left(\nu\left(x_{2}\right)\left(v_{1}, v_{2}\right), A\left(v_{3}\right)\right)= & \nu\left(\alpha\left(x_{2}\right)\right)\left(\nu\left(x_{1}\right)\left(v_{1}, v_{2}\right), A\left(v_{3}\right)\right) .
\end{aligned}
$$

Proof. The quadruple $(V ; \rho, \nu, A)$ is a generalized representation if and only if $[\pi+\bar{\rho}+\bar{\nu}, \pi+\bar{\rho}+\bar{\nu}]^{3 H L}=0$. By straightforward computations,

$$
[\pi+\bar{\rho}+\bar{\nu}, \pi+\bar{\rho}+\bar{\nu}]^{3 H L}\left(x_{1}, x_{2}, x_{3}, x_{4}, v\right)=0
$$

is equivalent to 2.2$)$; and

$$
[\pi+\bar{\rho}+\bar{\nu}, \pi+\bar{\rho}+\bar{\nu}]^{3 H L}\left(x_{1}, v, x_{2}, x_{3}, x_{4}\right)=0
$$


is equivalent to (2.3). Other identities can be proved similarly. The details are omitted.

Remark 2.6. By 2.2 and (2.3), the map $\rho$ in a generalized representation $(V ; \rho, \nu, A)$ gives rise to a usual representation in the sense of Definition 1.5. Conversely, for any representation $\rho,(V ; \rho, \nu=0, A)$ is a generalized representation.

DeFinition 2.7. Let $\left(V_{1} ; \rho_{1}, \nu_{1}, A_{1}\right)$ and $\left(V_{2} ; \rho_{2}, \nu_{2}, A_{2}\right)$ be two generalized representations of a 3 -Hom-Lie algebra $(\mathfrak{g},[\cdot, \cdot, \cdot], \alpha)$. They are said to be equivalent if there exists an isomorphism of vector spaces $T: V_{1} \rightarrow V_{2}$ such that

$T \rho_{1}(x, y)(u)=\rho_{2}(x, y)(T u), T \nu_{1}(x)(u, v)=\nu_{2}(x)(T u, T v), T \circ A_{1}=A_{2} \circ T$

for all $x, y \in \mathfrak{g}, u, v \in V_{1}$. In terms of diagrams, we have
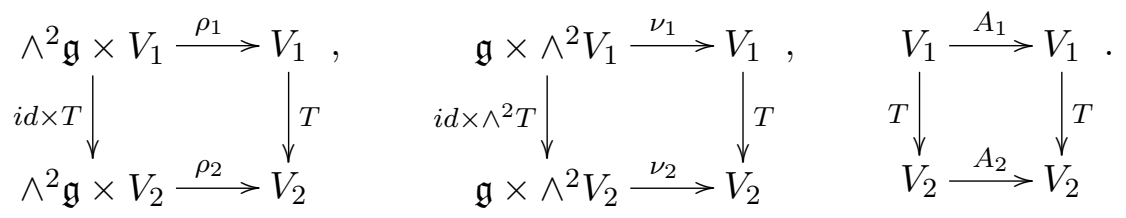

In the following, we provide a series of examples to illustrate the new concept of generalized representation and also a procedure to twist a generalized representation along linear maps.

EXAmPle 2.8. Let $\mathfrak{g}$ be an abelian 3-Hom-Lie algebra. Define $\rho=0$, and $\nu=\xi \otimes \pi$, where $\xi \in \mathfrak{g}^{*}$ and $\pi \in \operatorname{Hom}\left(\wedge^{2} V \otimes V\right)$ is a Hom-Lie algebra structure on $(V, \pi, A)$. Then $(V ; \rho, \nu, A)$ is a generalized representation. In fact, since $\mathfrak{g}$ is abelian and $\rho=0,(2.2)-(2.5)$ hold naturally. Since $\pi$ satisfies the Hom-Jacobi identity, (2.6) and (2.7) also hold.

Proposition 2.9. Let $(\mathfrak{g},[\cdot, \cdot, \cdot], \alpha)$ be a 3 -Hom-Lie algebra, $(V, \rho, \nu, A)$ be a generalized representation, $\beta: \mathfrak{g} \rightarrow \mathfrak{g}$ be an algebra morphism and $B: V \rightarrow V$ a linear map such that

$$
\begin{aligned}
B \circ \rho\left(x_{1}, x_{2}\right) & =\rho\left(\beta\left(x_{1}\right), \beta\left(x_{2}\right)\right) \circ B, \\
B \circ \nu(x) & =\nu(\beta(x)) \circ(B \otimes B), \\
B \circ A & =A \circ B .
\end{aligned}
$$


Then $(V, \widetilde{\rho}, \widetilde{\nu}, B)$ is a generalized representation of 3 -Hom-Lie algebra $(\mathfrak{g}$, $\left.[\cdot, \cdot, \cdot]_{\beta}, \beta \circ \alpha\right)$ where

$$
[\cdot, \cdot, \cdot]_{\beta}=[\cdot, \cdot, \cdot] \circ \beta^{\otimes 3}, \quad \widetilde{\rho}=B \circ \rho, \widetilde{\nu}(x)=B \circ \nu(x) .
$$

Proof. We have to show that $\widetilde{\rho}$ and $\widetilde{\nu}$ satisfy Eqs. $(2.2)-(2.7)$.

Let $x_{1}, x_{2}, x_{3} \in \mathfrak{g}$ and $v_{1}, v_{2} \in V$,

$$
\begin{aligned}
& \widetilde{\rho}\left(\beta \circ \alpha\left(x_{1}\right), \beta \circ \alpha\left(x_{2}\right)\right) \widetilde{\nu}\left(x_{3}\right)\left(v_{1}, v_{2}\right)-\widetilde{\nu}\left(\left[x_{1}, x_{2}, x_{3}\right]_{\beta}\right)\left(B \circ A\left(v_{1}\right), B \circ A\left(v_{2}\right)\right) \\
&-\widetilde{\nu}\left(\beta \circ \alpha\left(x_{3}\right)\right)\left(\widetilde{\rho}\left(x_{1}, x_{2}\right) v_{1}, B \circ A\left(v_{2}\right)\right) \\
& \quad-\widetilde{\nu}\left(\beta \circ \alpha\left(x_{3}\right)\right)\left(B \circ A\left(v_{2}\right), \text { widetilde } \rho\left(x_{1}, x_{2}\right) v_{1}\right) \\
&= B \circ \rho\left(\beta \circ \alpha\left(x_{1}\right), \beta \circ \alpha\left(x_{2}\right)\right) \circ B \circ \nu\left(x_{3}\right)\left(v_{1}, v_{2}\right) \\
&-B \circ \nu\left(\beta \circ\left[x_{1}, x_{2}, x_{3}\right]\right) \circ(B \otimes B)\left(A\left(v_{1}\right), A\left(v_{2}\right)\right) \\
&-B \circ \nu\left(\beta \circ \alpha\left(x_{3}\right)\right)\left(B \circ \rho\left(x_{1}, x_{2}\right) v_{1}, B \circ A\left(v_{2}\right)\right) \\
&\left.-B \circ \nu\left(\beta \alpha\left(x_{3}\right)\right)\left(B \circ A\left(v_{2}\right), B \circ \rho\left(x_{1}, x_{2}\right) v_{1}\right)\right) \\
&= B^{2} \circ\left(\rho\left(\alpha\left(x_{1}\right), \alpha\left(x_{2}\right)\right) \circ \nu\left(x_{3}\right)\left(v_{1}, v_{2}\right)-\nu\left(\left[x_{1}, x_{2}, x_{3}\right]\right)\left(A\left(v_{1}\right), A\left(v_{2}\right)\right)\right. \\
&\left.-\nu(\alpha(x))\left(\rho\left(x_{1}, x_{2}\right) v_{1}, A\left(v_{2}\right)\right)-\nu\left(\alpha\left(x_{3}\right)\right)[2 p t]\left(A\left(v_{2}\right), \rho\left(x_{1}, x_{2}\right) v_{1}\right)\right)=0, \\
&-\widetilde{\nu}\left(\beta \circ \alpha\left(x_{2}\right)\right)\left(\widetilde{\rho}\left(x_{3}, x_{1}\right) v_{1}, B \circ A\left(v_{2}\right)\right) \\
&-\widetilde{\rho}\left(\beta \circ \alpha\left(x_{2}\right), \beta \circ \alpha\left(x_{3}\right)\right) \widetilde{\nu}\left(x_{1}\right)\left(v_{1}, v_{2}\right) \\
&= B \circ \nu\left(\beta \circ \alpha\left(x_{1}\right)\right)\left(B \circ A\left(v_{1}\right), B \circ \rho\left(x_{2}, x_{3}\right) v_{2}\right) \\
&-B \circ \nu\left(\beta \circ \alpha\left(x_{3}\right)\right)\left(B \circ A\left(v_{2}\right), B \circ \rho\left(x_{2}, x_{1}\right) v_{1}\right) \\
&-B \circ \nu\left(\beta \circ \alpha\left(x_{2}\right)\right)\left(B \circ \rho\left(x_{3}, x_{1}\right) v_{1}, B \circ A\left(v_{2}\right)\right) \\
&-B \circ \rho\left(\beta \circ \alpha\left(x_{2}\right), \beta \circ \alpha\left(x_{3}\right)\right) \circ B \circ \nu\left(x_{1}\right)\left(v_{1}, v_{2}\right) \\
&= B \circ\left(\nu\left(\alpha\left(x_{1}\right)\right)\left(A\left(v_{1}\right), \rho\left(x_{2}, x_{3}\right) v_{2}\right)-\nu\left(\alpha\left(x_{3}\right)\right)\left(v_{2}, \rho\left(x_{2}, x_{1}\right) v_{1}\right)\right. \\
&\left.-\nu\left(\alpha\left(x_{2}\right)\right)\left(\rho\left(x_{3}, x_{1}\right) v_{1}, A\left(v_{2}\right)\right)-\rho\left(\alpha\left(x_{2}\right), \alpha\left(x_{3}\right)\right) \circ \nu\left(x_{1}\right)\left(v_{1}, v_{2}\right)\right)=0 .
\end{aligned}
$$

Then identities (2.4) and (2.5) are proved. One similarly proves identities (2.6) and (2.7).

Corollary 2.10. Let $(\mathfrak{g},[\cdot, \cdot, \cdot])$ be a 3 -Lie algebra, $(V, \rho, \nu)$ be a generalized representation, $\alpha: \mathfrak{g} \rightarrow \mathfrak{g}$ be an algebra morphism and $A: V \rightarrow V$ be a 
linear map such that for all $x_{1}, x_{2} \in \mathfrak{g}$ and $v_{1}, v_{2} \in V$,

$$
\begin{aligned}
A \circ \rho\left(x_{1}, x_{2}\right) & =\rho\left(\alpha\left(x_{1}\right), \alpha\left(x_{2}\right)\right) \circ A, \\
A \circ \nu(x)\left(v_{1}, v_{2}\right) & =\nu(\alpha(x)) \circ(A \otimes A)\left(v_{1}, v_{2}\right) .
\end{aligned}
$$

Then $(V, \widetilde{\rho}:=A \circ \rho, \widetilde{\nu}:=A \circ \nu, A)$ is a generalized representation of the 3 -Hom-Lie algebra $\left(L,[\cdot, \cdot, \cdot]_{\alpha}:=\alpha \circ[\cdot, \cdot, \cdot], \alpha\right)$.

EXAMPLE 2.11. Let $\mathfrak{g}$ be the 3-dimensional 3-Lie algebra defined with respect to a basis $\left\{e_{1}, e_{2}, e_{3}\right\}$ by the skew-symmetric bracket $\left[e_{1}, e_{2}, e_{3}\right]=e_{1}$. Let $V$ be a 2 -dimensional vector space and $\left\{v_{1}, v_{2}\right\}$ its basis. We have a representation defined by the following maps $(\rho, \mu)$, given with respect to previous bases by

$$
\begin{array}{rll}
\rho\left(e_{1}, e_{2}\right)\left(v_{1}\right)=0, & \rho\left(e_{1}, e_{2}\right)\left(v_{2}\right)=v_{1}, & \rho\left(e_{1}, e_{3}\right)\left(v_{1}\right)=0, \\
\rho\left(e_{1}, e_{3}\right)\left(v_{2}\right)=r_{1} v_{1}, & \rho\left(e_{2}, e_{3}\right)\left(v_{1}\right)=v_{1}, & \rho\left(e_{2}, e_{3}\right)\left(v_{2}\right)=r_{2} v_{1}, \\
\nu\left(e_{1}\right)\left(v_{1}, v_{2}\right)=0, & \nu\left(e_{2}\right)\left(v_{1}, v_{2}\right)=s v_{1}, & \nu\left(e_{3}\right)\left(v_{1}, v_{2}\right)=s r_{1} v_{1},
\end{array}
$$

where $r_{1}, r_{2}, s$ are parameters in $\mathbb{K}$.

Let $\alpha: \mathfrak{g} \rightarrow \mathfrak{g}$ be an algebra morphism and $A \in g l(V)$ defined respectively by:

$$
\begin{aligned}
\alpha\left(e_{1}\right)=\lambda e_{1}, & \alpha\left(e_{2}\right)=e_{2}, \quad \alpha\left(e_{3}\right)=e_{3}, \\
A\left(v_{1}\right)=\lambda v_{1}, & A\left(v_{2}\right)=r_{2} v_{1}+v_{2},
\end{aligned}
$$

where $\lambda$ is a parameter in $\mathbb{K}$. They satisfy

$$
\begin{aligned}
A \circ \rho\left(x_{1}, x_{2}\right) & =\rho\left(\alpha\left(x_{1}\right), \alpha\left(x_{2}\right)\right) \circ A, \\
A \circ \nu(x)\left(u_{1}, u_{2}\right) & =\nu(\alpha(x)) \circ(A \otimes A)\left(u_{1}, u_{2}\right),
\end{aligned}
$$

where $x, x_{1}, x_{2}$ are in $\mathfrak{g}$ and $u_{1}, u_{2}$ in $V$.

Then, using the Twist procedure, $(V ; \widetilde{\rho}, \widetilde{\nu}, A)$ is a generalized representation of the 3 -Hom-Lie algebra $\left(\mathfrak{g},[\cdot, \cdot, \cdot]_{\alpha}, \alpha\right)$. More precisely, we have

$$
\begin{array}{rlrl}
{\left[e_{1}, e_{2}, e_{3}\right]_{\alpha}} & =\left[\alpha\left(e_{1}\right), \alpha\left(e_{2}\right), \alpha\left(e_{3}\right)\right]=\lambda e_{1}, \\
\widetilde{\rho}\left(e_{1}, e_{2}\right)\left(v_{1}\right)=0, & \widetilde{\rho}\left(e_{1}, e_{2}\right)\left(v_{2}\right)=\lambda v_{1}, \\
\widetilde{\rho}\left(e_{1}, e_{3}\right)\left(v_{1}\right)=0, & \widetilde{\rho}\left(e_{1}, e_{3}\right)\left(v_{2}\right)=r_{1} r_{2}(\lambda-1) v_{1}-r_{1} v_{2}, \\
\widetilde{\rho}\left(e_{2}, e_{3}\right)\left(v_{1}\right)=\lambda v_{1}, & \widetilde{\rho}\left(e_{2}, e_{3}\right)\left(v_{2}\right)=r_{2} \lambda v_{1}, \\
\widetilde{\nu}\left(e_{1}\right)\left(v_{1}, v_{2}\right)=0, & \widetilde{\nu}\left(e_{2}\right)\left(v_{1}, v_{2}\right) & =s \lambda v_{1}, \quad \widetilde{\nu}\left(e_{3}\right)\left(v_{1}, v_{2}\right)=s r_{1} \lambda v_{1} .
\end{array}
$$


EXAMPLE 2.12. Let $\mathfrak{g}$ be the 4-dimensional 3-Lie algebra defined, with respect to a basis $\left\{e_{1}, e_{2}, e_{3}, e_{4}\right\}$, by the skew-symmetric brackets

$$
\left[e_{1}, e_{2}, e_{4}\right]=e_{3}, \quad\left[e_{1}, e_{3}, e_{4}\right]=e_{2}, \quad\left[e_{2}, e_{3}, e_{4}\right]=e_{1} .
$$

Every generalized representation $(V ; \rho, \nu)$, on a 2 -dimensional vector space $V$ with trivial $\rho$, of $\mathfrak{g}$ is given by one of the following maps $\nu$ defined, with respect to a basis $\left\{v_{1}, v_{2}\right\}$ of $V$, by

$$
\begin{array}{ll}
\nu\left(e_{1}\right)\left(v_{1}, v_{2}\right)=0, & \nu\left(e_{2}\right)\left(v_{1}, v_{2}\right)=0, \\
\nu\left(e_{3}\right)\left(v_{1}, v_{2}\right)=0, & \nu\left(e_{4}\right)\left(v_{1}, v_{2}\right)=s_{1} v_{1}+s_{2} v_{2},
\end{array}
$$

where $s_{1}, s_{2}$ are parameters in $\mathbb{K}$, and $s_{1} s_{2} \neq 0$.

Let $\alpha: \mathfrak{g} \rightarrow \mathfrak{g}$ be a 3-Lie algebra morphism and $A: V \rightarrow V$ be a linear map, defined respectively by

$$
\begin{gathered}
\alpha\left(e_{1}\right)=a_{1} e_{1}, \quad \alpha\left(e_{2}\right)=-a_{1} e_{2}, \quad \alpha\left(e_{3}\right)=a_{1} e_{3}, \quad \alpha\left(e_{4}\right)=\frac{-1}{a_{1}} e_{4}, \\
A\left(v_{1}\right)=-a_{1} v_{1}, \quad A\left(v_{2}\right)=a_{2} v_{1}+\frac{a_{2} s_{2}-a_{1} s_{1}}{s_{1}} v_{2},
\end{gathered}
$$

where $a_{1}, a_{2}, s_{1}, s_{2}$ are parameters in $\mathbb{K}$ such that, $a_{1} s_{1} s_{2} \neq 0$.

They satisfy $A \circ \nu(x)=\nu(\alpha(x)) \circ(A \otimes A)$. Therefore, using the Twist procedure, $(V ; \widetilde{\rho}, \widetilde{\nu}, A)$ is a generalized representation of the 3-Hom-Lie algebra $\left(L,[\cdot, \cdot, \cdot]_{\alpha}, \alpha\right)$ with trivial $\widetilde{\rho}$. Namely, we have

$$
\left[e_{1}, e_{2}, e_{4}\right]_{\alpha}=a_{1} e_{3}, \quad\left[e_{1}, e_{3}, e_{4}\right]_{\alpha}=-a_{1} e_{2}, \quad\left[e_{2}, e_{3}, e_{4}\right]_{\alpha}=a_{1} e_{1},
$$

and

$$
\begin{aligned}
& \widetilde{\nu}\left(e_{1}\right)\left(v_{1}, v_{2}\right)=0, \quad \widetilde{\nu}\left(e_{2}\right)\left(v_{1}, v_{2}\right)=0, \quad \widetilde{\nu}\left(e_{3}\right)\left(v_{1}, v_{2}\right)=0, \\
& \widetilde{\nu}\left(e_{4}\right)\left(v_{1}, v_{2}\right)=\left(a_{2} s_{2}-a_{1} s_{1}\right) v_{1}+\left(\frac{a_{2} s_{2}^{2}}{s_{1}}-a_{1} s_{2}\right) v_{2} .
\end{aligned}
$$

\section{New COHOMOlOGy COMPlex of 3-Hom-Lie Algebras}

Based on the generalized representations defined in the previous section, we introduce a new type of cohomology for 3-Hom-Lie algebras.

Let $(\mathfrak{g},[\cdot, \cdot, \cdot], \alpha)$ be a 3 -Hom-Lie algebra and $(V ; \rho, \nu, A)$ be a generalized representation of $\mathfrak{g}$. We set $\widetilde{C}_{\alpha+A, A}^{p}(\mathfrak{g} \oplus V, V)$ to be the set of $(p+1)$-Homcochains, which are defined as a subset of $C_{\alpha+A, A}^{p}(\mathfrak{g} \oplus V, V)$ such that

$$
C_{\alpha+A, A}^{p}(\mathfrak{g} \oplus V, V)=\widetilde{C}_{\alpha+A, A}^{p}(\mathfrak{g} \oplus V, V) \oplus C_{A}^{p}(V, V) .
$$


Elements of $C_{\alpha+A, A}^{p}(\mathfrak{g} \oplus V, V)$ are of the form

$$
\varphi: \wedge^{2}(\mathfrak{g} \oplus V) \otimes(p \text { times }) \otimes \wedge^{2}(\mathfrak{g} \oplus V) \wedge(\mathfrak{g} \oplus V) \rightarrow V .
$$

By direct calculation, we have

$$
\left[\pi+\bar{\rho}+\bar{\nu}, \widetilde{C}_{\alpha+A, A}^{\bullet}(\mathfrak{g} \oplus V, V)\right] \subseteq \widetilde{C}_{\alpha+A, A}^{\bullet+1}(\mathfrak{g} \oplus V, V) .
$$

Define $d: \widetilde{C}_{\alpha+A, A}^{p}(\mathfrak{g} \oplus V, V) \rightarrow \widetilde{C}_{\alpha+A, A}^{p+1}(\mathfrak{g} \oplus V, V)$ by

$$
d(\varphi):=[\pi+\bar{\rho}+\bar{\nu}, \varphi]^{3 H L}, \quad \varphi \in \widetilde{C}_{\alpha+A, A}^{p}(\mathfrak{g} \oplus V, V) .
$$

Theorem 3.1. Let $(V ; \rho, \nu, A)$ be a generalized representation of a 3 -Hom-Lie algebra $\mathfrak{g}$. Then $d \circ d=0$. Thus, we obtain a new cohomology complex, where the space of $p$-Hom-cochains is given by $\widetilde{C}_{\alpha+A, A}^{p-1}(\mathfrak{g} \oplus V, V)$.

Proof. By the graded Jacobi identity, for any $\varphi \in \widetilde{C}_{\alpha+A, A}^{p-1}(\mathfrak{g} \oplus V, V)$, one obtains

$$
\begin{aligned}
d \circ d(\varphi): & =\left[\pi+\bar{\rho}+\bar{\nu},[\pi+\bar{\rho}+\bar{\nu}, \varphi]^{3 H L}\right]^{3 H L} \\
& =\frac{1}{2}\left[[\pi+\bar{\rho}+\bar{\nu}, \pi+\bar{\rho}+\bar{\nu}]^{3 H L}, \varphi\right]^{3 H L}=0 .
\end{aligned}
$$

An element $\varphi \in \widetilde{C}_{\alpha+A, A}^{p-1}(\mathfrak{g} \oplus V, V)$ is called a $p$-cocycle if $d(\varphi)=0$; it is called a $p$-coboundary if there exists $f \in \widetilde{C}_{\alpha+A, A}^{p-2}(\mathfrak{g} \oplus V, V)$ such that $\varphi=d(f)$.

Denote by $\mathcal{Z}_{3 H L}^{p}(\mathfrak{g} ; V)$ and $\mathcal{B}_{3 H L}^{p}(\mathfrak{g} ; V)$ the sets of $p$-cocycles and $p$ coboundaries respectively. By Theorem 3.1, we have $\mathcal{B}_{3 H L}^{p}(\mathfrak{g} ; V) \subset \mathcal{Z}^{p}(\mathfrak{g} ; V)$. We define the $p$-th cohomolgy group $\mathcal{H}_{3 H L}^{p}(\mathfrak{g} ; V)$ to be $\mathcal{Z}_{3 H L}^{p}(\mathfrak{g} ; V) / \mathcal{B}_{3 H L}^{p}(\mathfrak{g} ; V)$.

The following proposition provides a relationship between this new cohomology and the one given by (1.5).

Proposition 3.2. There is a forgetful map from $\mathcal{H}_{3 H L}^{p}(\mathfrak{g} ; V)$ to $H_{3 H L}^{p}(\mathfrak{g} ; V)$.

Proof. It is obvious that $C_{\alpha, A}^{p}(\mathfrak{g}, V) \subseteq \widetilde{C}_{\alpha+A, A}^{p}(\mathfrak{g} \oplus V, V)$. By direct calculation, for $X_{i} \in \wedge^{2} \mathfrak{g}, z \in \mathfrak{g}$, we have

$$
d(\varphi)\left(X_{1}, \ldots, X_{p+1}, z\right)=\delta_{\rho}(\varphi)\left(X_{1}, \ldots, X_{p+1}, z\right), \quad \varphi \in C_{\alpha, A}^{p}(\mathfrak{g}, V),
$$

where $\delta_{\rho}$ is the coboundary operator given by (1.4). Thus, the natural projection from $\widetilde{C}_{\alpha+A, A}^{p}(\mathfrak{g} \oplus V, V)$ to $C_{\alpha, A}^{p}(\mathfrak{g}, V)$ induces a forgetful map from $\mathcal{H}_{3 H L}^{p}(\mathfrak{g} ; V)$ to $H_{3 H L}^{p}(\mathfrak{g} ; V)$. 
In the sequel, we give some characterization of low dimensional cocycles.

Proposition 3.3. A linear map $\varphi \in \operatorname{Hom}(\mathfrak{g}, V)$ is a 1-cocycle if only if for all $x_{1}, x_{2}, x_{3} \in \mathfrak{g}, v \in V$, the following identities hold:

$$
\begin{aligned}
\varphi \circ \alpha & =A \circ \varphi, \\
\nu\left(x_{1}\right)\left(\varphi\left(x_{2}\right), v\right)-\nu\left(x_{2}\right)\left(\varphi\left(x_{1}\right), v\right) & =0, \\
\varphi\left(\left[x_{1}, x_{2}, x_{3}\right]\right)-\rho\left(\alpha\left(x_{1}\right), \alpha\left(x_{2}\right)\right)\left(\varphi\left(x_{3}\right)\right) & \\
-\rho\left(\alpha\left(x_{2}\right), \alpha\left(x_{3}\right)\right)\left(\varphi\left(x_{1}\right)\right)-\rho\left(\alpha\left(x_{3}\right), \alpha\left(x_{1}\right)\right)\left(\varphi\left(x_{2}\right)\right) & =0 .
\end{aligned}
$$

Proof. For $\varphi \in \operatorname{Hom}(\mathfrak{g}, V)$ satisfying $\varphi \circ \alpha=A \circ \varphi$, we have

$$
d(\varphi)\left(x_{1}, x_{2}, v\right)=\nu\left(x_{1}\right)\left(\varphi\left(x_{2}\right), v\right)-\nu\left(x_{2}\right)\left(\varphi\left(x_{1}\right), v\right),
$$

and

$$
\begin{aligned}
d(\varphi)\left(x_{1}, x_{2}, x_{3}\right)= & \delta_{\rho}(\varphi)\left(x_{1}, x_{2}, x_{3}\right) \\
= & \rho\left(\alpha\left(x_{1}\right), \alpha\left(x_{2}\right)\right)\left(\varphi\left(x_{3}\right)\right)+\rho\left(\alpha\left(x_{2}\right), \alpha\left(x_{3}\right)\right)\left(\varphi\left(x_{1}\right)\right) \\
& +\rho\left(\alpha\left(x_{3}\right), \alpha\left(x_{1}\right)\right)\left(\varphi\left(x_{2}\right)\right)-\varphi\left(\left[x_{1}, x_{2}, x_{3}\right]\right) .
\end{aligned}
$$

Proposition 3.4. A 2-cochain $\varphi_{1}+\varphi_{2}+\varphi_{3} \in \widetilde{C}_{\alpha, A}^{1}(\mathfrak{g} \oplus V, V)$, where $\varphi_{1} \in \operatorname{Hom}\left(\wedge^{2} V \wedge \mathfrak{g}, V\right), \varphi_{2} \in \operatorname{Hom}\left(\wedge^{2} \mathfrak{g} \wedge V, V\right), \varphi_{3} \in \operatorname{Hom}\left(\wedge^{3} \mathfrak{g}, V\right)$, is a 2cocycle if and only if for all $x_{i} \in \mathfrak{g}, v_{j} \in V$ and $v \in V$, the following identities hold:

$$
\begin{aligned}
0= & -\rho\left(\alpha\left(x_{1}\right), \alpha\left(x_{2}\right)\right)\left(\varphi_{3}\left(x_{3}, x_{4}, x_{5}\right)\right)-\varphi_{3}\left(\alpha\left(x_{1}\right), \alpha\left(x_{2}\right),\left[x_{3}, x_{4}, x_{5}\right]\right) \\
& +\rho\left(\alpha\left(x_{4}\right), \alpha\left(x_{5}\right)\right)\left(\varphi_{3}\left(x_{1}, x_{2}, x_{3}\right)\right)+\varphi_{3}\left(\left[x_{1}, x_{2}, x_{3}\right], \alpha\left(x_{4}\right), \alpha\left(x_{5}\right)\right) \\
& +\rho\left(\alpha\left(x_{5}\right), \alpha\left(x_{3}\right)\right)\left(\varphi_{3}\left(x_{1}, x_{2}, x_{4}\right)\right)+\varphi_{3}\left(\alpha\left(x_{3}\right),\left[x_{1}, x_{2}, x_{4}\right], \alpha\left(x_{5}\right)\right) \\
& +\rho\left(\alpha\left(x_{3}\right), \alpha\left(x_{4}\right)\right)\left(\varphi_{3}\left(x_{1}, x_{2}, x_{5}\right)\right)+\varphi_{3}\left(\alpha\left(x_{3}\right), \alpha\left(x_{4}\right),\left[x_{1}, x_{2}, x_{5}\right]\right), \\
0= & \nu\left(\alpha\left(x_{4}\right)\right)\left(\varphi_{3}\left(x_{1}, x_{2}, x_{3}\right), A(v)\right)+\nu\left(\alpha\left(x_{3}\right)\right)\left(A(v), \varphi_{3}\left(x_{1}, x_{2}, x_{4}\right)\right) \\
& +\rho\left(\alpha\left(x_{1}\right), \alpha\left(x_{2}\right)\right)\left(\varphi_{2}\left(x_{3}, x_{4}, v\right)\right)-\rho\left(\alpha\left(x_{3}\right), \alpha\left(x_{4}\right)\right)\left(\varphi_{2}\left(x_{1}, x_{2}, v\right)\right) \\
& -\varphi_{2}\left(\left[x_{1}, x_{2}, x_{3}\right], \alpha\left(x_{4}\right), A(v)\right)-\varphi_{2}\left(\alpha\left(x_{3}\right),\left[x_{1}, x_{2}, x_{4}\right], A(v)\right), \\
0= & \left.\nu\left(\alpha\left(x_{1}\right)\right)\left(A(v), \varphi_{3}\left(x_{2}, x_{3}, x_{4}\right)\right)+\rho\left(x_{3}\right), \alpha\left(x_{4}\right)\right)\left(\varphi_{2}\left(x_{1}, x_{2}, v\right)\right) \\
& -\rho\left(\alpha\left(x_{2}\right), \alpha\left(x_{4}\right)\right)\left(\varphi_{2}\left(x_{1}, x_{3}, v\right)\right)+\rho\left(\alpha\left(x_{2}\right), \alpha\left(x_{3}\right)\right)\left(\varphi_{2}\left(x_{1}, x_{4}, v\right)\right) \\
& +\varphi_{2}\left(\alpha\left(x_{3}\right), \alpha\left(x_{4}\right), \rho\left(x_{1}, x_{2}\right)(v)\right)-\varphi_{2}\left(\alpha\left(x_{2}\right), \alpha\left(x_{4}\right), \rho\left(x_{1}, x_{3}\right)(v)\right) \\
& +\varphi_{2}\left(\alpha\left(x_{2}\right), \alpha\left(x_{3}\right), \rho\left(x_{1}, x_{4}\right)(v)\right)-\varphi_{2}\left(\alpha\left(x_{1}\right),\left[x_{2}, x_{3}, x_{4}\right], A(v)\right),
\end{aligned}
$$




$$
\begin{aligned}
& 0=\nu\left(\alpha\left(x_{3}\right)\right)\left(A\left(v_{2}\right), \varphi_{2}\left(x_{1}, x_{2}, v_{1}\right)\right)+\nu\left(\alpha\left(x_{3}\right)\right)\left(\varphi_{2}\left(x_{1}, x_{2}, A\left(v_{2}\right)\right), v_{1}\right) \\
& +\varphi_{2}\left(\alpha\left(x_{1}\right), \alpha\left(x_{2}\right), \nu\left(x_{3}\right)\left(v_{1}, v_{2}\right)\right)+\rho\left(\alpha\left(x_{1}\right), \alpha\left(x_{2}\right)\right)\left(\varphi_{1}\left(v_{1}, v_{2}, x_{3}\right)\right) \\
& -\varphi_{1}\left(\rho\left(x_{1}, x_{2}\right)\left(v_{1}\right), A\left(v_{2}\right), \alpha\left(x_{3}\right)\right)-\varphi_{1}\left(A\left(v_{1}\right), \rho\left(x_{1}, x_{2}\right)\left(v_{2}\right), \alpha\left(x_{3}\right)\right) \\
& -\varphi_{1}\left(A\left(v_{1}\right), A\left(v_{2}\right),\left[x_{1}, x_{2}, x_{3}\right]\right) \text {, } \\
& 0=\nu\left(\alpha\left(x_{3}\right)\right)\left(A\left(v_{2}\right), \varphi_{2}\left(x_{2}, x_{1}, v_{1}\right)\right)+\nu\left(\alpha\left(x_{2}\right)\right)\left(\varphi_{2}\left(x_{3}, x_{1}, v_{1}\right), A\left(v_{2}\right)\right) \\
& -\nu\left(\alpha\left(x_{1}\right)\right)\left(A\left(v_{1}\right), \varphi_{2}\left(x_{2}, x_{3}, v_{2}\right)\right)+\varphi_{2}\left(\alpha\left(x_{2}\right), \alpha\left(x_{3}\right), \nu\left(x_{1}\right)\left(v_{1}, v_{2}\right)\right) \\
& +\rho\left(\alpha\left(x_{2}\right), \alpha\left(x_{3}\right)\right)\left(\varphi_{1}\left(v_{1}, v_{2}, x_{1}\right)\right)+\varphi_{1}\left(\rho\left(x_{1}, x_{2}\right)\left(v_{1}\right), A\left(v_{2}\right), \alpha\left(x_{3}\right)\right) \\
& -\varphi_{1}\left(A\left(v_{1}\right), \rho\left(x_{2}, x_{3}\right)\left(v_{2}\right), \alpha\left(x_{1}\right)\right)+\varphi_{1}\left(A\left(v_{2}\right), \rho\left(x_{1}, x_{3}\right)\left(v_{1}\right), \alpha\left(x_{2}\right)\right) \text {, } \\
& 0=\varphi_{2}\left(\alpha\left(x_{1}\right), \alpha\left(x_{3}\right), \nu\left(x_{2}\right)\left(v_{1}, v_{2}\right)\right)-\varphi_{2}\left(\alpha\left(x_{2}\right), \alpha\left(x_{3}\right), \nu\left(x_{1}\right)\left(v_{1}, v_{2}\right)\right) \\
& -\varphi_{2}\left(\alpha\left(x_{1}\right), \alpha\left(x_{2}\right), \nu\left(x_{3}\right)\left(v_{1}, v_{2}\right)\right)+\rho\left(\alpha\left(x_{1}\right), \alpha\left(x_{3}\right)\right)\left(\varphi_{1}\left(v_{1}, v_{2}, x_{2}\right)\right) \\
& -\rho\left(\alpha\left(x_{1}\right), \alpha\left(x_{2}\right)\right)\left(\varphi_{1}\left(v_{1}, v_{2}, x_{3}\right)\right)-\rho\left(\alpha\left(x_{2}\right), \alpha\left(x_{3}\right)\right)\left(\varphi_{1}\left(v_{1}, v_{2}, x_{1}\right)\right) \\
& +\varphi_{1}\left(A\left(v_{1}\right), A\left(v_{2}\right),\left[x_{1}, x_{2}, x_{3}\right]\right) \text {, } \\
& 0=-\nu\left(\alpha\left(x_{2}\right)\right)\left(\varphi_{1}\left(v_{1}, v_{2}, x_{1}\right), A\left(v_{3}\right)\right)-\nu\left(\alpha\left(x_{2}\right)\right)\left(A\left(v_{2}\right), \varphi_{1}\left(v_{1}, v_{3}, x_{1}\right)\right) \\
& +\nu\left(\alpha\left(x_{1}\right)\right)\left(A\left(v_{1}\right), \varphi_{1}\left(v_{2}, v_{3}, x_{2}\right)\right)-\varphi_{1}\left(\nu\left(x_{1}\right)\left(v_{1}, v_{2}\right), A\left(v_{3}\right), \alpha\left(x_{2}\right)\right) \\
& -\varphi_{1}\left(A\left(v_{2}\right), \nu\left(x_{1}\right)\left(v_{1}, v_{3}\right), \alpha\left(x_{2}\right)\right)+\varphi_{1}\left(A\left(v_{1}\right), \nu\left(x_{2}\right)\left(v_{2}, v_{3}\right), \alpha\left(x_{1}\right)\right) \text {, } \\
& 0=\nu\left(\alpha\left(x_{2}\right)\right)\left(\varphi_{1}\left(v_{1}, v_{2}, x_{1}\right), A\left(v_{3}\right)\right)-\nu\left(\alpha\left(x_{1}\right)\right)\left(A\left(v_{3}\right), \varphi_{1}\left(v_{1}, v_{2}, x_{2}\right)\right) \\
& +\varphi_{1}\left(\nu\left(x_{1}\right)\left(v_{1}, v_{2}\right), A\left(v_{3}\right), \alpha\left(x_{2}\right)\right)-\varphi_{1}\left(\nu\left(x_{2}\right)\left(v_{1}, v_{2}\right), A\left(v_{3}\right), \alpha\left(x_{1}\right)\right) \text {. }
\end{aligned}
$$

Proof. For $\varphi_{3} \in \operatorname{Hom}\left(\wedge^{3} \mathfrak{g}, V\right)$, we have

$$
\begin{aligned}
d\left(\varphi_{3}\right)\left(x_{1}, x_{2}, x_{3}, x_{4}, x_{5}\right)= & \rho\left(\alpha\left(x_{1}\right), \alpha\left(x_{2}\right)\right)\left(\varphi_{3}\left(x_{3}, x_{4}, x_{5}\right)\right) \\
& +\varphi_{3}\left(\alpha\left(x_{1}\right), \alpha\left(x_{2}\right),\left[x_{3}, x_{4}, x_{5}\right]\right) \\
& -\rho\left(\alpha\left(x_{4}\right), \alpha\left(x_{5}\right)\right)\left(\varphi_{3}\left(x_{1}, x_{2}, x_{3}\right)\right) \\
& -\varphi_{3}\left(\left[x_{1}, x_{2}, x_{3}\right], \alpha\left(x_{4}\right), \alpha\left(x_{5}\right)\right) \\
& -\rho\left(\alpha\left(x_{5}\right), \alpha\left(x_{3}\right)\right)\left(\varphi_{3}\left(x_{1}, x_{2}, x_{4}\right)\right) \\
& -\varphi_{3}\left(\alpha\left(x_{3}\right),\left[x_{1}, x_{2}, x_{4}\right], \alpha\left(x_{5}\right)\right) \\
& -\rho\left(\alpha\left(x_{3}\right), \alpha\left(x_{4}\right)\right)\left(\varphi_{3}\left(x_{1}, x_{2}, x_{5}\right)\right) \\
& -\varphi_{3}\left(\alpha\left(x_{3}\right), \alpha\left(x_{4}\right),\left[x_{1}, x_{2}, x_{5}\right]\right), \\
d\left(\varphi_{3}\right)\left(x_{1}, x_{2}, x_{3}, x_{4}, v\right)= & \nu\left(\alpha\left(x_{4}\right)\right)\left(\varphi_{3}\left(x_{1}, x_{2}, x_{3}\right), A(v)\right) \\
& +\nu\left(\alpha\left(x_{3}\right)\right)\left(A(v), \varphi_{3}\left(x_{1}, x_{2}, x_{4}\right)\right), \\
d\left(\varphi_{3}\right)\left(x_{1}, v, x_{2}, x_{3}, x_{4}\right)= & \nu\left(\alpha\left(x_{1}\right)\right)\left(A(v), \varphi_{3}\left(x_{2}, x_{3}, x_{4}\right)\right) .
\end{aligned}
$$


For $\varphi_{2} \in \operatorname{Hom}\left(\wedge^{2} \mathfrak{g} \wedge V, V\right)$, we have

$$
\begin{aligned}
d\left(\varphi_{2}\right)\left(x_{1}, x_{2}, x_{3}, x_{4}, v\right)= & \rho\left(\alpha\left(x_{1}\right), \alpha\left(x_{2}\right)\right)\left(\varphi_{2}\left(x_{3}, x_{4}, v\right)\right) \\
& -\rho\left(\alpha\left(x_{3}\right), \alpha\left(x_{4}\right)\right)\left(\varphi_{2}\left(x_{1}, x_{2}, v\right)\right) \\
& -\varphi_{2}\left(\left[x_{1}, x_{2}, x_{3}\right], \alpha\left(x_{4}\right), A(v)\right) \\
& -\varphi_{2}\left(\alpha\left(x_{3}\right),\left[x_{1}, x_{2}, x_{4}\right], A(v)\right), \\
d\left(\varphi_{2}\right)\left(x_{1}, v, x_{2}, x_{3}, x_{4}\right)= & \rho\left(\alpha\left(x_{3}\right), \alpha\left(x_{4}\right)\right)\left(\varphi_{2}\left(x_{1}, x_{2}, v\right)\right) \\
& -\rho\left(\alpha\left(x_{2}\right), \alpha\left(x_{4}\right)\right)\left(\varphi_{2}\left(x_{1}, x_{3}, v\right)\right) \\
& +\rho\left(\alpha\left(x_{2}\right), \alpha\left(x_{3}\right)\right)\left(\varphi_{2}\left(x_{1}, x_{4}, v\right)\right) \\
& +\varphi_{2}\left(\alpha\left(x_{3}\right), \alpha\left(x_{4}\right), \rho\left(x_{1}, x_{2}\right)(v)\right) \\
& -\varphi_{2}\left(\alpha\left(x_{2}\right), \alpha\left(x_{4}\right), \rho\left(x_{1}, x_{3}\right)(v)\right) \\
& +\varphi_{2}\left(\alpha\left(x_{2}\right), \alpha\left(x_{3}\right), \rho\left(x_{1}, x_{4}\right)(v)\right) \\
& -\alpha_{2}\left(x_{1},\left[x_{2}, x_{3}, x_{4}\right], v\right), \\
d\left(\varphi_{2}\right)\left(x_{1}, x_{2}, v_{1}, v_{2}, x_{3}\right)= & \nu\left(\alpha\left(x_{3}\right)\right)\left(A\left(v_{2}\right), \varphi_{2}\left(x_{1}, x_{2}, v_{1}\right)\right) \\
& +\nu\left(\alpha\left(x_{3}\right)\right)\left(\varphi_{2}\left(x_{1}, x_{2}, v_{2}\right), A\left(v_{1}\right)\right) \\
& +\varphi_{2}\left(\alpha\left(x_{1}\right), \alpha\left(x_{2}\right), \nu\left(x_{3}\right)\left(v_{1}, v_{2}\right)\right), \\
d\left(\varphi_{2}\right)\left(x_{1}, v_{1}, x_{2}, v_{2}, x_{3}\right)= & \nu\left(\alpha\left(x_{3}\right)\left(A\left(v_{2}\right), \varphi_{2}\left(x_{2}, x_{1}, v_{1}\right)\right)\right. \\
& +\nu\left(\alpha\left(x_{2}\right)\right)\left(\varphi_{2}\left(x_{3}, x_{1}, v_{1}\right), A\left(v_{2}\right)\right) \\
& -\nu\left(\alpha\left(x_{1}\right)\right)\left(A\left(v_{1}\right), \varphi_{2}\left(x_{2}, x_{3}, v_{2}\right)\right) \\
& +\varphi_{2}\left(\alpha\left(x_{2}\right), \alpha\left(x_{3}\right), \nu\left(x_{1}\right)\left(v_{1}, v_{2}\right)\right), \\
d\left(\varphi_{2}\right)\left(v_{1}, v_{2}, x_{1}, x_{2}, x_{3}\right)= & \varphi_{2}\left(\alpha\left(x_{1}\right), \alpha\left(x_{3}\right), \nu\left(x_{2}\right)\left(v_{1}, v_{2}\right)\right) \\
& -\varphi_{2}\left(\alpha\left(x_{2}\right), \alpha\left(x_{3}\right), \nu\left(x_{1}\right)\left(v_{1}, v_{2}\right)\right) \\
& -\varphi_{2}\left(\alpha\left(x_{1}\right), \alpha\left(x_{2}\right), \nu\left(x_{3}\right)\left(v_{1}, v_{2}\right)\right) .
\end{aligned}
$$

For $\varphi_{1} \in \operatorname{Hom}\left(\wedge^{2} V \wedge \mathfrak{g}, V\right)$, we have

$$
\begin{aligned}
d\left(\varphi_{1}\right)\left(x_{1}, x_{2}, v_{1}, v_{2}, x_{3}\right)= & \rho\left(\alpha\left(x_{1}\right), \alpha\left(x_{2}\right)\right)\left(\varphi_{1}\left(v_{1}, v_{2}, x_{3}\right)\right) \\
& -\varphi_{1}\left(\rho\left(x_{1}, x_{2}\right)\left(v_{1}\right), A\left(v_{2}\right), \alpha\left(x_{3}\right)\right) \\
& -\varphi_{1}\left(A\left(v_{1}\right), \rho\left(x_{1}, x_{2}\right)\left(v_{2}\right), \alpha\left(x_{3}\right)\right) \\
& -\varphi_{1}\left(A\left(v_{1}\right), A\left(v_{2}\right),\left[x_{1}, x_{2}, x_{3}\right]\right), \\
d\left(\varphi_{1}\right)\left(x_{1}, v_{1}, x_{2}, v_{2}, x_{3}\right)= & \rho\left(\alpha\left(x_{2}\right), \alpha\left(x_{3}\right)\right)\left(\varphi_{1}\left(v_{1}, v_{2}, x_{1}\right)\right) \\
& +\varphi_{1}\left(\rho\left(x_{1}, x_{2}\right)\left(v_{1}\right), A\left(v_{2}\right), \alpha\left(x_{3}\right)\right) \\
& -\varphi_{1}\left(A\left(v_{1}\right), \rho\left(x_{2}, x_{3}\right)\left(v_{2}\right), \alpha\left(x_{1}\right)\right) \\
& +\varphi_{1}\left(A\left(v_{2}\right), \rho\left(x_{1}, x_{3}\right)\left(v_{1}\right), \alpha\left(x_{2}\right)\right),
\end{aligned}
$$




$$
\begin{aligned}
d\left(\varphi_{1}\right)\left(v_{1}, v_{2}, x_{1}, x_{2}, x_{3}\right)= & \rho\left(\alpha\left(x_{1}\right), \alpha\left(x_{3}\right)\right)\left(\varphi_{1}\left(v_{1}, v_{2}, x_{2}\right)\right) \\
& -\rho\left(\alpha\left(x_{1}\right), \alpha\left(x_{2}\right)\right)\left(\varphi_{1}\left(v_{1}, v_{2}, x_{3}\right)\right) \\
& -\rho\left(\alpha\left(x_{2}\right), \alpha\left(x_{3}\right)\right)\left(\varphi_{1}\left(v_{1}, v_{2}, x_{1}\right)\right) \\
& +\varphi_{1}\left(A\left(v_{1}\right), A\left(v_{2}\right),\left[x_{1}, x_{2}, x_{3}\right]\right), \\
d\left(\varphi_{1}\right)\left(x_{1}, v_{1}, v_{2}, v_{3}, x_{2}\right)= & -\nu\left(\alpha\left(x_{2}\right)\right)\left(\varphi_{1}\left(v_{1}, v_{2}, x_{1}\right), A\left(v_{3}\right)\right) \\
& -\nu\left(\alpha\left(x_{2}\right)\right)\left(A\left(v_{2}\right), \varphi_{1}\left(v_{1}, v_{3}, x_{1}\right)\right) \\
& +\nu\left(\alpha\left(x_{1}\right)\right)\left(A\left(v_{1}\right), \varphi_{1}\left(v_{2}, v_{3}, x_{2}\right)\right) \\
& -\varphi_{1}\left(\nu\left(x_{1}\right)\left(v_{1}, v_{2}\right), A\left(v_{3}\right), \alpha\left(x_{2}\right)\right) \\
& -\varphi_{1}\left(A\left(v_{2}\right), \nu\left(x_{1}\right)\left(v_{1}, v_{3}\right), \alpha\left(x_{2}\right)\right) \\
& +\varphi_{1}\left(A\left(v_{1}\right), \nu\left(x_{2}\right)\left(v_{2}, v_{3}\right), \alpha\left(x_{1}\right)\right), \\
d\left(\varphi_{1}\right)\left(v_{1}, v_{2}, x_{1}, x_{2}, v_{3}\right)= & \nu\left(\alpha\left(x_{2}\right)\right)\left(\varphi_{1}\left(v_{1}, v_{2}, x_{1}\right), A\left(v_{3}\right)\right) \\
& -\nu\left(\alpha\left(x_{1}\right)\right)\left(A\left(v_{3}\right), \varphi_{1}\left(v_{1}, v_{2}, x_{2}\right)\right) \\
& +\varphi_{1}\left(\nu\left(x_{1}\right)\left(v_{1}, v_{2}\right), A\left(v_{3}\right), \alpha\left(x_{2}\right)\right) \\
& -\varphi_{1}\left(\nu\left(x_{2}\right)\left(v_{1}, v_{2}\right), A\left(v_{3}\right), \alpha\left(x_{1}\right)\right) .
\end{aligned}
$$

Thus, $d\left(\varphi_{1}+\varphi_{2}+\varphi_{3}\right)=0$ if and only if Eqs. (3.1)-(3.8) hold.

In the following we provide an example of computation of 2-cocycles of a 3-dimensional 3-Hom-Lie algebra.

EXAmPLE 3.5. Let $(\mathfrak{g},[\cdot, \cdot, \cdot], \alpha)$ be the 3-dimensional 3-Hom-Lie algebra defined, with respect to a basis $\left\{e_{1}, e_{2}, e_{3}\right\}$, by $\left[e_{1}, e_{2}, e_{3}\right]=a_{1} e_{1}, \alpha\left(e_{1}\right)=a_{1} e_{1}$, $\alpha\left(e_{2}\right)=a_{2} e_{2}, \alpha\left(e_{3}\right)=\frac{1}{a_{2}} e_{3}$. Let $V$ be a 2-dimensional vector space, $\left\{v_{1}, v_{2}\right\}$ its basis and $A \in g l(V)$ defined by: $A\left(v_{1}\right)=a_{1} v_{1}, A\left(v_{2}\right)=\frac{a_{2} a_{3}}{a_{1}} v_{2}$, where $a_{1}, a_{2}, a_{3}$ are parameters in $\mathbb{K}$.

We consider the generalized representation $(V, \rho, \nu, A)$, where $\rho$ and $\nu$ are defined with respect to the basis by

$$
\begin{array}{cll}
\rho\left(e_{1}, e_{2}\right)\left(v_{1}\right)=0, & \rho\left(e_{1}, e_{2}\right)\left(v_{2}\right)=0, & \rho\left(e_{1}, e_{3}\right)\left(v_{1}\right)=0, \\
\rho\left(e_{1}, e_{3}\right)\left(v_{2}\right)=r_{2} a_{1} v_{1}, & \rho\left(e_{2}, e_{3}\right)\left(v_{1}\right)=a_{1} v_{1}, & \rho\left(e_{2}, e_{3}\right)\left(v_{2}\right)=\frac{r_{1} a_{2} a_{3}}{a_{1}} v_{2}, \\
\nu\left(e_{1}\right)\left(v_{1}, v_{2}\right)=0, & \nu\left(e_{2}\right)\left(v_{1}, v_{2}\right)=0, & \nu\left(e_{3}\right)\left(v_{1}, v_{2}\right)=s_{1} a_{1} v_{1},
\end{array}
$$

with $s_{1}, r_{1}, r_{2}$ parameters in $\mathbb{K}$ and $a_{1} a_{2} s_{1} \neq 0$. 
We have the following 2-cocycles: $\varphi_{1}=0, \varphi_{3}=0$ and $\varphi_{2}$ defined as

$$
\begin{array}{ll}
\varphi_{2}\left(e_{1}, e_{2}, v_{1}\right)=0, & \varphi_{2}\left(e_{1}, e_{2}, v_{2}\right)=0, \\
\varphi_{2}\left(e_{1}, e_{3}, v_{1}\right)=c_{1} v_{1}, & \varphi_{2}\left(e_{1}, e_{3}, v_{2}\right)=0, \\
\varphi_{2}\left(e_{2}, e_{3}, v_{1}\right)=c_{2} v_{1}, & \varphi_{2}\left(e_{2}, e_{3}, v_{2}\right)=c_{3} v_{2},
\end{array}
$$

where $c_{1}, c_{2}, c_{3}$ are parameters in $\mathbb{K}$.

\section{Abelian extensions of 3-Hom-Lie algebras}

In this section, we show that associated to any abelian extension, there is a generalized representation and a 2-cocycle.

Definition 4.1. Let $\left(\mathfrak{g},[\cdot, \cdot, \cdot]_{\mathfrak{g}}, \alpha\right),\left(V,[\cdot, \cdot, \cdot]_{V}, A\right)$ and $\left(\hat{\mathfrak{g}},[\cdot, \cdot, \cdot]_{\hat{\mathfrak{g}}}, \alpha_{\hat{\mathfrak{g}}}\right)$ be 3 -Hom-Lie algebras and $i: V \rightarrow \hat{\mathfrak{g}}, p: \hat{\mathfrak{g}} \rightarrow \mathfrak{g}$ be morphisms of 3-HomLie algebras. The following sequence of 3 -Hom-Lie algebras is a short exact sequence if $\operatorname{Im}(i)=\operatorname{Ker}(p), \operatorname{Ker}(i)=0$ and $\operatorname{Im}(p)=\mathfrak{g}$ :

$$
0 \rightarrow V \stackrel{i}{\longrightarrow} \hat{\mathfrak{g}} \stackrel{p}{\longrightarrow} \mathfrak{g} \rightarrow 0,
$$

where $A(V)=\alpha_{\hat{\mathfrak{g}}}(V)$. In this case, we call $\hat{\mathfrak{g}}$ an extension of $\mathfrak{g}$ by $V$, and denote it by $E_{\hat{\mathfrak{g}}}$. It is called an abelian extension if $V$ is an abelian ideal of $\hat{\mathfrak{g}}$, i.e., $[u, v, w]_{V}=0$ for all $u, v, w \in V$. A section $\sigma$ of $p: \hat{\mathfrak{g}} \rightarrow \mathfrak{g}$ consists of a linear maps $\sigma: \mathfrak{g} \rightarrow \hat{\mathfrak{g}}$ such that $p \circ \sigma=i d_{\mathfrak{g}}$ and $\sigma \circ \alpha=\alpha_{\hat{\mathfrak{g}}} \circ \sigma$.

Definition 4.2. Two extensions of 3-Hom-Lie algebras,

$$
E_{\hat{\mathfrak{g}}}: 0 \rightarrow V \stackrel{i_{1}}{\longrightarrow} \hat{\mathfrak{g}} \stackrel{p_{1}}{\longrightarrow} \mathfrak{g} \rightarrow 0 \quad \text { and } \quad E_{\tilde{\mathfrak{g}}}: 0 \rightarrow V \stackrel{i_{2}}{\longrightarrow} \tilde{\mathfrak{g}} \stackrel{p_{2}}{\longrightarrow} \mathfrak{g} \rightarrow 0
$$

are equivalent if there exists a morphism of 3-Hom-Lie algebras $\phi: \hat{\mathfrak{g}} \rightarrow \tilde{\mathfrak{g}}$ such that the following diagram commutes:

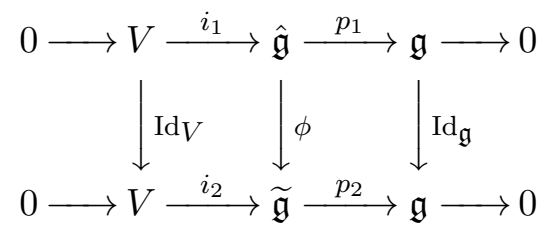

A linear map $\sigma: \mathfrak{g} \rightarrow \hat{\mathfrak{g}}$ is called a splitting of $\mathfrak{g}$ if it satisfies $p \circ \sigma=i d_{\mathfrak{g}}$. If there exists a splitting which is also a homomorphism between 3-Hom-Lie 
algebras, we say that the abelian extension is split. Let $\hat{\mathfrak{g}}$ be a split abelian extension and $\sigma: \mathfrak{g} \rightarrow \hat{\mathfrak{g}}$ the corresponding splitting. Define $\rho: \wedge^{2} \mathfrak{g} \rightarrow \mathfrak{g l}(V)$ and $\nu: \mathfrak{g} \rightarrow \operatorname{Hom}\left(\wedge^{2} V, V\right)$ by

$$
\begin{aligned}
& \rho(x, y)(u)=[\sigma(x), \sigma(y), u]_{\hat{\mathfrak{g}}}, \\
& \nu(x)(u, v)=[\sigma(x), u, v]_{\hat{\mathfrak{g}}} .
\end{aligned}
$$

Then, we can transfer the 3-Hom-Lie algebra structure on $\hat{\mathfrak{g}}$ to that on $\mathfrak{g} \oplus V$ in terms of $\rho$ and $\nu$.

Note that the Hom-Filippov-Jacobi identity gives the character of $\rho$ and $\nu$ :

$$
\begin{aligned}
{[x+u, y+v, z+w]_{(\rho, \nu)}=} & {[x, y, z]+\rho(x, y)(w)+\rho(y, z)(u)+\rho(z, x)(v) } \\
& +\nu(x)(v \wedge w)+\nu(y)(w \wedge u)+\nu(z)(u \wedge v) .
\end{aligned}
$$

However, by Theorem 2.4, it is straightforward to obtain the following proposition.

Proposition 4.3. Any split abelian extension of 3-Hom-Lie algebras is isomorphic to a generalized semidirect of product 3-Hom-Lie algebra.

Now, for non-split abelian extensions, we can further define $\omega: \wedge^{3} \mathfrak{g} \rightarrow V$ by

$$
\omega(x, y, z)=[\sigma(x), \sigma(y), \sigma(z)]_{\mathfrak{g}}-\sigma[x, y, z]_{\mathfrak{g}} .
$$

Then, we also transfer the 3 -Hom-Lie algebra structure on $\hat{\mathfrak{g}}$ to that on $\mathfrak{g} \oplus V$ in terms of $\rho, \nu$ and $\omega$ :

$$
\begin{aligned}
{\left[x_{1}+v_{1}, x_{2}+v_{2}, x_{3}+v_{3}\right]_{(\rho, \nu, \omega)}=} & \left.x_{1}, x_{2}, x_{3}\right]_{\mathfrak{g}}+\rho\left(x_{1}, x_{2}\right)\left(v_{3}\right) \\
& +\rho\left(x_{3}, x_{1}\right)\left(v_{2}\right)+\rho\left(x_{2}, x_{3}\right)\left(v_{1}\right) \\
& +\nu\left(x_{1}\right)\left(v_{2}, v_{3}\right)+\nu\left(x_{2}\right)\left(v_{3}, v_{1}\right) \\
& +\nu\left(x_{3}\right)\left(v_{1}, v_{2}\right)+\omega\left(x_{1}, x_{2}, x_{3}\right) .
\end{aligned}
$$

Theorem 4.4. With above notations, $\left(\mathfrak{g} \oplus V,[\cdot, \cdot, \cdot]_{(\rho, \nu, \omega)}, \alpha_{\mathfrak{g} \oplus V)}\right)$ is a 3 Hom-Lie algebra if and only if for all $x_{1}, x_{2}, x_{3}, x_{4}, x_{5} \in \mathfrak{g}$ and $v, v_{1}, v_{2}, v_{3} \in V$, Eqs. (2.4)-(2.7) and the following identities hold: 


$$
\begin{aligned}
0= & -\rho\left(\alpha\left(x_{1}\right), \alpha\left(x_{2}\right)\right)\left(\omega\left(x_{3}, x_{4}, x_{5}\right)\right)-\omega\left(\alpha\left(x_{1}\right), \alpha\left(x_{2}\right),\left[x_{3}, x_{4}, x_{5}\right]\right) \\
& +\rho\left(\alpha\left(x_{4}\right), \alpha\left(x_{5}\right)\right)\left(\omega\left(x_{1}, x_{2}, x_{3}\right)\right)+\omega\left(\left[x_{1}, x_{2}, x_{3}\right], \alpha\left(x_{4}\right), \alpha\left(x_{5}\right)\right) \\
& +\rho\left(\alpha\left(x_{5}\right), \alpha\left(x_{3}\right)\right)\left(\omega\left(x_{1}, x_{2}, x_{4}\right)\right)+\omega\left(\alpha\left(x_{3}\right),\left[x_{1}, x_{2}, x_{4}\right], \alpha\left(x_{5}\right)\right) \\
& +\rho\left(\alpha\left(x_{3}\right), \alpha\left(x_{4}\right)\right)\left(\omega\left(x_{1}, x_{2}, x_{5}\right)\right)+\omega\left(\alpha\left(x_{3}\right), \alpha\left(x_{4}\right),\left[x_{1}, x_{2}, x_{5}\right]\right), \\
0= & \nu\left(\alpha\left(x_{1}\right)\right)\left(A(v), \omega\left(x_{2}, x_{3}, x_{4}\right)\right)+\rho\left(\left[x_{2}, x_{3}, x_{4}\right], \alpha\left(x_{1}\right)\right)(A(v)) \\
& +\rho\left(\alpha\left(x_{3}\right), \alpha\left(x_{4}\right)\right) \rho\left(x_{1}, x_{2}\right)(v)-\rho\left(\alpha\left(x_{2}\right), \alpha\left(x_{4}\right)\right) \rho\left(x_{1}, x_{3}\right)(v) \\
& +\rho\left(\alpha\left(x_{2}\right), \alpha\left(x_{3}\right)\right) \rho\left(x_{1}, x_{4}\right)(v), \\
0= & \rho\left(\alpha\left(x_{1}\right), \alpha\left(x_{2}\right)\right) \rho\left(x_{3}, x_{4}\right)(v)-\rho\left(\alpha\left(x_{3}\right), \alpha\left(x_{4}\right)\right) \rho\left(x_{1}, x_{2}\right)(v) \\
& -\rho\left(\left[x_{1}, x_{2}, x_{3}\right], \alpha\left(x_{4}\right)\right)(A(v))-\nu\left(\alpha\left(x_{4}\right)\right)\left(A(v), \omega\left(x_{1}, x_{2}, x_{3}\right)\right) \\
& -\rho\left(\alpha\left(x_{3}\right),\left[x_{1}, x_{2}, x_{4}\right]\right)(A(v))+\nu\left(\alpha\left(x_{3}\right)\right)\left(A(v), \omega\left(x_{1}, x_{2}, x_{4}\right)\right) .
\end{aligned}
$$

The Fundamental Identity gives the character of $\rho, \nu$ and $\omega$.

Proof. The triple $\left(\mathfrak{g} \oplus V,[\cdot, \cdot, \cdot]_{(\rho, \nu, \omega)}, \alpha_{\mathfrak{g} \oplus V}\right)$ defines a 3 -Hom-Lie algebra if and only if the Hom-Filippov-Jacobi identity holds on all elements of $\mathfrak{g} \oplus$ $V$. Condition (4.1) is obtained using the Hom-Filippov-Jacobi identity on $\left\{x_{1}, x_{2}, x_{3}, x_{4}, x_{5}\right\}$ elements of $\mathfrak{g}$.

Similarly, elements $\left\{x_{1}, v, x_{2}, x_{3}, x_{4}\right\}$ gives Eq. (4.2), $\left\{x_{1}, x_{2}, v, x_{3}, x_{4}\right\}$ gives Eq. (4.3), $\left\{x_{1}, x_{2}, v_{1}, v_{2}, x_{3}\right\}$ gives Eq. (2.4), $\left\{v_{1}, x_{1}, v_{2}, x_{2}, x_{3}\right\}$ gives Eq. 2.5), $\left\{v_{1}, x_{1}, v_{2}, v_{3}, x_{2}\right\}$ gives Eq. (2.6) and $\left\{v_{1}, v_{2}, v_{3}, x_{1}, x_{2}\right\}$ gives Eq. 2.7.

Conversely, if Eqs. (2.4)-(2.7) and Eqs. (4.1)-(4.3) hold, it is straightforward to see that for all $e_{1}, \ldots, e_{5} \in \mathfrak{g} \oplus V$, the Hom-Filippov-Jacobi identity holds. Thus, $\left(\mathfrak{g} \oplus V,[\cdot, \cdot, \cdot \cdot]_{(\rho, \nu, \omega)}, \alpha_{\mathfrak{g} \oplus V}\right)$ is a 3-Hom-Lie algebra.

Remark 4.5. The triple $(\rho, \nu, \omega)$ is a cochain with respect to the new cohomology defined in Section 3 but it is not necessarily a 2-cocycle.

\section{ACKNowledgements}

The authors would like to thank Yunhe Sheng for his comments and suggestions. 


\section{REFERENCES}

[1] F. Ammar, S. Mabrouk, A. Makhlouf, Representations and cohomology of $n$-ary multiplicative Hom-Nambu-Lie algebras, J. Geom. Phys. $61(10)(2011), 1898-1913$.

[2] J. Arnlind, A. Makhlouf, S. Silvestrov, Construction of $n$-Lie algebras and $n$-ary Hom-Nambu-Lie algebras, J. Math. Phys. 52(12) (2011), 123502, 13pp.

[3] J. Arnlind, A. Kitouni, A. Makhlouf, S. Silvestrov, Structure and cohomology of 3-Lie algebras induced by Lie algebras, in "Algebra, Geometry and Mathematical Physics", Springer Proc. Math. Stat. 85, Springer, Heidelberg, 2014, 123-144.

[4] H. Ataguema, A. Makhlouf, S. Silvestrov, Generalization of $n$-ary Nambu algebras and beyond, J. Math. Phys. 50 (8) (2009), 083501, 15 pp.

[5] J. BAGGer, N. LAMBERT, Gauge symmetry and supersymmetry of multiple M2-branes, Phys. Rev. D 77 (6) (2008), 065008, 6 pp.

[6] J. Bagger, N. Lambert, Three-algebras and $\mathscr{N}=6$ Chern-Simons gauge theories, Phys. Rev. D $\mathbf{7 9}$ (2) (2009), 025002, 8 pp.

[7] R. BAI, C. BAI, J. Wang, Realizations of 3-Lie algebras, J. Math. Phys. 51 (6) (2010), 063505, 12 pp.

[8] R. Bai, G. Song, Y. Zhang, On classification of $n$-Lie algebras, Front. Math. China 6 (4) (2011), 581-606.

[9] A. Basu, J.A. Harvey, The M2-M5 brane system and a generalized Nahm's equation, Nuclear Phys. B $\mathbf{7 1 3}$ (1-3) (2005), 136-150.

[10] L. CAI, Y. SHEng, Hom-big brackets: theory and applications, SIGMA Symmetry Integrability Geom. Methods Appl. 12 (2016), Paper No. 014, $18 \mathrm{pp}$.

[11] Y. Daletskit, L. Takhtajan, Leibniz and Lie algebra structures for Nambu algebra, Lett. Math. Phys. 39 (2) (1997), 127-141.

[12] J.A. DE AzCÁRRAGA, J.M. IzQUiERdo, $n$-ary algebras: a review with applications, J. Phys. A: Math. Theor. 43 (2010), 293001.

[13] J.A. DE AzCÁRRAGA, J.M. IzQUiERdo, Cohomology of Filippov algebras and an analogue of Whitehead's lemma, J. Phys. Conf. Ser. 175 (2009), 012001.

[14] J. Figueroa-O'Farrill, Deformations of 3-algebras, J. Math. Phys. 50 (11) (2009), 113514, 27 pp.

[15] V.T. Filippov, n-Lie algebras (Russian), Sibirsk. Mat. Zh. 26 (6) (1985) $126-140$.

[16] Y. FrÉGIER, Non-abelian cohomology of extensions of Lie algebras as Deligne groupoid, J. Algebra 398 (2014), 243-257.

[17] P. Gautheron, Some remarks concerning Nambu mechanics, Lett. Math. Phys. 37 (1) (1996), 103-116.

[18] J. Hartwig, D. Larsson, S. Silvestrov, Deformations of Lie algebras using $\sigma$-derivations, J. Algebra 295 (2) (2006), 314-361. 
[19] J. Liu, A. Makhlouf, Y. Sheng, A new approach to representations of 3-Lie algebras and abelian extensions, Algebr. Represent. Theory 20 (6) (2017), 1415-1431.

[20] P. Ho, R. Hou, Y. Matsuo, Lie 3-algebra and multiple M2-branes, J. High Energy Phys. 2008 (6) (2008), 020, 30 pp.

[21] Sh.M. Kasymov, On a theory of $n$-Lie algebras (Russian), Algebra i Logika 26 (3) (1987), $277-297$.

[22] A. Makhlouf, On Deformations of $n$-Lie Algebras, in "Non-Associative and Non-Commutative Algebra and Operator Theory", Springer Proc. Math. Stat., 160, Springer, Cham, 2016, 55-81.

[23] Y. Nambu, Generalized Hamiltonian dynamics, Phys. Rev. D 7 (1973), $2405-2412$.

[24] A. Nijenhuis, R. Richardson, Cohomology and Deformations in Graded Lie Algebras, Bull. Amer. Math. Soc. 72 (1966), 1-29.

[25] Y. Sheng, Representations of hom-Lie algebras, Algebr. Represent. Theory 15 (6) (2012), 1081-1098.

[26] L. Song, A. Makhlouf, R. Tang, On non-abelian extensions of 3-Lie algebras, Commun. Theor. Phys. 69 (4) (2018), 347-356.

[27] G. Papadopoulos, M2-branes, 3-Lie algebras and Plücker relations, J. High Energy Phys. 2008 (5), 054, 9 pp.

[28] M. Rotkiewicz, Cohomology ring of $n$-Lie algebras, Extracta Math. 20 (3) (2005), $219-232$.

[29] L. TAKhtajan, On foundation of the generalized Nambu mechanics, Comm. Math. Phys. 160 (2) (1994), 295-315.

[30] L. TAKhtajan, A higher order analog of Chevalley-Eilenberg complex and deformation theory of $n$-algebras, St. Petersburg Math. J. 6 (2) (1995), 429 438.

[31] J. ZhaO, L. Chen, n-ary Hom-Nambu algebras, arXiv:1505.08168v1, 2015. 